\title{
The Mean Square Error of a random fuzzy vector based on the support function and the Steiner point ${ }^{\text {th }}$
}

\author{
Beatriz Sinova ${ }^{a, b}$, Sara de la Rosa de Sáa ${ }^{a}$, María Rosa Casals ${ }^{a}$, \\ María Ángeles Gil ${ }^{\mathrm{a}}$, Antonia Salas*a \\ ${ }^{a}$ Departamento de Estadística e I.O. y D.M., \\ Universidad de Oviedo, 33071 Oviedo, Spain \\ ${ }^{b}$ Department of Applied Mathematics, Computer Science and Statistics, \\ Ghent University, 9000 Gent, Belgium
}

\begin{abstract}
Metrics between fuzzy values are a topic with interest for different purposes. Among them, statistics with fuzzy data is growing in modelling and techniques largely through the use of suitable distances between such data. This paper introduces a generalized (actually, parameterized) $L^{2}$ metric between fuzzy vectors which is based on their representation in terms of their support function and Steiner points. Consequently, the metric takes into account the deviation in 'central location' (represented by the Steiner points) and the deviation in 'shape' (represented by a deviation defined in terms of the support function and Steiner points). Then, sufficient conditions can be given for this representation to characterize fuzzy vectors, which is valuable for different aims, like optimization studies. Properties of the metric are analyzed and its application to quantify the mean (square) error of a fuzzy value in estimating the value of a fuzzy vector-valued random element is examined. Some immediate implications from this mean error are finally described.
\end{abstract}

Keywords: fuzzy vector, mean square error, random fuzzy vector, Steiner point, support function

\footnotetext{
This paper has been prepared in honor to our friend Françesc Esteva on the occasion of his (SOMEWHAT ELDERLY) birthday. Although we have never worked directly on his research field, his contributions to fuzzy logic have certainly benefit our own.

*corresponding author: antonia@uniovi.es, Fax: (+34) 985103354.
} 


\section{Introduction}

Distances between fuzzy sets are a topic which has received a deep attention in the literature. On one hand, it has been considered in connection with studies of similarity between fuzzy sets (see, for instance, Dubois et al. [14], Beg and Ashraf [2], and Esteva et al. [15]). On the other hand, for statistical purposes such as classification of fuzzy-valued elements or inferential statistics with fuzzy set-valued random elements, distances play a key role (see, for instance, Blanco-Fernández et al. $[4,5]$ ).

In several papers (e.g., Bertoluzza et al. [3], Casals et al. [7], Trutschnig et al. [33]) the suitability of considering metrics between fuzzy vector values taking into account both their 'central location' along with their 'shape' has been pointed out and discussed.

For some practical goals, often related to optimization tasks, it can also be convenient to use metrics based on representations of fuzzy vector values for which there exist sufficient conditions characterizing fuzzy vectors.

A well-known distance satisfying the last desirable property is the metric $\rho_{2}$ (see Diamond and Kloeden [10]), which is based on the support function of the involved fuzzy vectors and takes into account the usual inner product for Hilbert space functional values. When fuzzy vectors reduce to fuzzy numbers, this metric corresponds to the one based on the infimum/supremum characterization of fuzzy numbers.

Recently (see Sinova et al. [28]), a new parameterized representation of fuzzy numbers, along with an associated $L^{2}$ metric, has been introduced. This representation describes each fuzzy number by means of an indicator of its central location and an indicator (in fact, two) of its shape, and there exist sufficient conditions characterizing fuzzy numbers. This is the essential difference with Bertoluzza et al.'s metric [3] (see also Trutschnig et al. [33]): there don't exist sufficient conditions for the mid-point/spread representation of fuzzy numbers behind such a metric characterizing fuzzy numbers.

This paper aims to extend the same idea behind this metric to the fuzzy vectorial-valued case. The rest of the paper is organized as follows: Section 2 recalls some basic concepts on fuzzy vectors and the suggested representation in terms of the support function and the generalized Steiner points; Section 3 introduces an $L^{2}$ metric based on the representation in Section 2 and its properties are examined; Section 4 states a measure of the mean square error based on the new metric, and some applications are indicated in Section 5 . 


\section{The representation of fuzzy vectors through their support function and generalized Steiner point}

Let $\mathcal{F}_{c}^{*}\left(\mathbb{R}^{p}\right)$ denote the space of (bounded) fuzzy vectors of $\mathbb{R}^{p}$, where a (bounded) fuzzy vector $\widetilde{U} \in \mathcal{F}_{c}^{*}\left(\mathbb{R}^{p}\right)$ is a fuzzy set of $\mathbb{R}^{p}$, that is, a mapping $\widetilde{U}: \mathbb{R}^{p} \rightarrow[0,1]$ such that it is normal and fuzzy-convex, upper semicontinuous and its support set is bounded. Equivalently, for all $\alpha \in[0,1]$ the $\alpha$-level of $\widetilde{U}$, defined as

$$
\widetilde{U}_{\alpha}= \begin{cases}\left\{\mathbf{x} \in \mathbb{R}^{p}: \widetilde{U}(\mathbf{x}) \geq \alpha\right\} & \text { if } \alpha \in(0,1] \\ \operatorname{cl}\left\{\mathbf{x} \in \mathbb{R}^{p}: \widetilde{U}(\mathbf{x})>0\right\} & \text { if } \alpha=0,\end{cases}
$$

is a nonempty compact subset of $\mathbb{R}^{p}$.

On the space $\mathcal{F}_{c}^{*}\left(\mathbb{R}^{p}\right)$ one can consider the usual fuzzy arithmetic based on Zadeh's extension principle [37]. Given $\widetilde{U}, \widetilde{V} \in \mathcal{F}_{c}^{*}\left(\mathbb{R}^{p}\right)$ and $\gamma \in \mathbb{R}$, the sum of $\widetilde{U}$ and $\widetilde{V}$ is defined as the fuzzy vector $\widetilde{U}+\widetilde{V} \in \mathcal{F}_{c}^{*}\left(\mathbb{R}^{p}\right)$ such that

$$
(\widetilde{U}+\widetilde{V})(\mathbf{t})=\sup _{\mathbf{y}+\mathbf{z}=\mathbf{t}} \min \{\widetilde{U}(\mathbf{y}), \widetilde{V}(\mathbf{z})\}
$$

or, equivalently and based on Nguyen [23], for each $\alpha \in[0,1]$ :

$$
(\widetilde{U}+\widetilde{V})_{\alpha}=\text { Minkowski sum of } \widetilde{U}_{\alpha} \text { and } \widetilde{V}_{\alpha}=\left\{\mathbf{y}+\mathbf{z}: \mathbf{y} \in \widetilde{U}_{\alpha}, \mathbf{z} \in \widetilde{V}_{\alpha}\right\} .
$$

The product of $\widetilde{U}$ by the scalar $\gamma$ is defined as the fuzzy vector $\gamma \cdot \widetilde{U} \in \mathcal{F}_{c}^{*}\left(\mathbb{R}^{p}\right)$ such that

$$
(\gamma \cdot \widetilde{U})(\mathbf{t})=\sup _{\mathbf{y} \in \mathbb{R}^{p}: \mathbf{y}=\gamma \mathbf{t}} \widetilde{U}(\mathbf{y})= \begin{cases}\widetilde{U}(\mathbf{t} / \gamma) & \text { if } \gamma \neq 0 \\ \mathbf{1}_{\{0\}}(\mathbf{t}) & \text { if } \gamma=0\end{cases}
$$

or, equivalently and based on Nguyen [23], for each $\alpha \in[0,1]$ :

$$
(\gamma \cdot \widetilde{U})_{\alpha}=\gamma \cdot \widetilde{U}_{\alpha}=\left\{\gamma \cdot \mathbf{y}: \mathbf{y} \in \widetilde{U}_{\alpha}\right\}
$$

It is well-known that when $\mathcal{F}_{c}^{*}\left(\mathbb{R}^{p}\right)$ is endowed with the two preceding operations we get a semi-linear but not a linear space, since $\widetilde{U}+(-1) \cdot \widetilde{U} \neq \mathbb{1}_{\{\mathbf{0}\}}$ (neutral element for the fuzzy sum), but in case $\widetilde{U}$ reduces to the indicator function of an element in $\mathbb{R}^{p}$. As a consequence of this, one cannot state 
an extension of the difference on the space $\mathcal{F}_{c}^{*}\left(\mathbb{R}^{p}\right)$ which can simultaneously be always well-defined and preserve the properties of the difference with real numbers in connection with the sum. This concern motivates and strongly reinforces the interest in having suitable metrics between fuzzy vectors, especially in developing certain statistics with them.

Elements in $\mathcal{F}_{c}^{*}\left(\mathbb{R}^{p}\right)$ can be characterized by means of their support function (see, for instance, Liang et al. [21]), which in accordance with the approach by Puri and Ralescu [24] is the function extending level-wise the notion of the support function of a set (see Castaing and Valadier [8]).

The support function of $\widetilde{U} \in \mathcal{F}_{c}^{*}\left(\mathbb{R}^{p}\right)$ is given by the mapping $s_{\widetilde{U}}$ : $[0,1] \times \mathbb{S}^{p-1} \rightarrow \mathbb{R}$ defined so that

$$
s_{\widetilde{U}}(\alpha, \mathbf{u})=\sup _{\mathbf{x} \in \widetilde{U}_{\alpha}}\langle\mathbf{u}, \mathbf{x}\rangle
$$

for all $\mathbf{u} \in \mathbb{S}^{p-1}, \alpha \in[0,1]$, where $\langle\cdot, \cdot\rangle$ denotes the inner (dot) product on $\mathbb{R}^{p}$ and $\mathbb{S}^{p-1}$ is the unit sphere in $\mathbb{R}^{p}$. In general, one can state that $s_{\widetilde{U}}(\alpha, \mathbf{u})$ represents the 'oriented' distance from $\mathbf{0} \in \mathbb{R}^{p}$ to the supporting hyperplane of $\widetilde{U}_{\alpha}$ which is orthogonal to $\mathbf{u}$.

It should be recalled (see, for instance, Butnariu et al. [6]) that a realvalued function $s:[0,1] \times \mathbb{S}^{p-1} \rightarrow \mathbb{R}$ is the support function of a unique fuzzy vector $\widetilde{U} \in \mathcal{F}_{c}^{*}\left(\mathbb{R}^{p}\right)$ (i.e., $s=s_{\widetilde{U}}$ ) if and only if $s$ satisfies that

$s . i)$ for all $\alpha \in[0,1], \mathbf{u}, \mathbf{v} \in \mathbb{S}^{p-1}$

$$
\|\mathbf{u}+\mathbf{v}\| \cdot s\left(\alpha, \frac{\mathbf{u}+\mathbf{v}}{\|\mathbf{u}+\mathbf{v}\|}\right) \leq s(\alpha, \mathbf{u})+s(\alpha, \mathbf{v})
$$

s.ii) $s(\cdot, \mathbf{u})$ is non-increasing, left-continuous on $(0,1]$ and right-continuous at 0 .

Actually, $\widetilde{U}$ is the fuzzy vector such that for all $\alpha \in[0,1]$ :

$$
\widetilde{U}_{\alpha}=\left\{\mathbf{x} \in \mathbb{R}^{p}:\langle\mathbf{u}, \mathbf{x}\rangle \leq s(\alpha, \mathbf{u}) \text { for all } \mathbf{u} \in \mathbb{S}^{p-1}\right\} .
$$

For purposes of quantifying a 'central location' of an element in $\mathcal{F}_{c}^{*}\left(\mathbb{R}^{p}\right)$, one can consider the well-known generalized Steiner point (or centroid) which has been defined (see, for instance, Diamond and Kloeden [11, 12], Körner [20], Diamond and Körner [13], Butnariu et al. [6], Vetterlein and Navara [34, 35], and Liang et al. [21]) by extending level-wise the Steiner points for convex sets (see Schneider [26]). 
Given a measure $\mu$ on the measurable space $\left([0,1], \mathcal{B}_{[0,1]}\right)$ that can be formalized by means of an absolutely continuous probability measure on $[0,1]$ (i.e., a measure for which there exists a nonnegative function $\eta$ which is defined, integrable and normalized on $[0,1]$, and such that $d \mu(\alpha)=\eta(\alpha) d \alpha)$, and $\lambda_{p}$ denoting the normalized Lebesgue measure on $\mathbb{S}^{p-1}$, the $\boldsymbol{\mu}$-Steiner point of $\widetilde{U} \in \mathcal{F}_{c}^{*}\left(\mathbb{R}^{p}\right)$, if it exists, is given by the vector value

$$
\mathbf{S}^{\mu}(\widetilde{U})=\iint_{[0,1] \times \mathbb{S}^{p-1}} \mathbf{u} \cdot s_{\widetilde{U}}(\alpha, \mathbf{u}) d \lambda_{p}(\mathbf{u}) d \mu(\alpha) .
$$

Then, on the basis of the results by Vetterlein and Navara [34, 35], any vectorial-valued function $\mathbf{S}: \mathcal{F}_{c}^{*}\left(\mathbb{R}^{p}\right) \rightarrow \mathbb{R}^{p}$ such that

S.i) $\mathbf{S}(\widetilde{U}) \in \widetilde{U}_{0}$,

S.ii) for all $\widetilde{U}, \widetilde{V} \in \mathcal{F}_{c}^{*}\left(\mathbb{R}^{p}\right)$ we have that $\mathbf{S}(\widetilde{U}+\widetilde{V})=\mathbf{S}(\widetilde{U})+\mathbf{S}(\widetilde{V})$,

S.iii) for all $\widetilde{U} \in \mathcal{F}_{c}^{*}\left(\mathbb{R}^{p}\right)$ and any Euclidean isometry $\tau$ of $\mathbb{R}^{p}$, i.e., $(\tau(\widetilde{U}))_{\alpha}$ $=\tau\left(\widetilde{U}_{\alpha}\right)$, we have that $\mathbf{S}(\tau(\widetilde{U}))=\tau(\mathbf{S}(\widetilde{U}))$,

$\mathbf{S} . i v$ ) $\mathbf{S}$ is continuous w.r.t. the supremum metric $d_{\infty}$ (and, hence, for most of the possible metrics that may be considered on $\left.\mathcal{F}_{c}^{*}\left(\mathbb{R}^{p}\right) \rightarrow \mathbb{R}^{p}\right)$,

satisfies that there exists an absolutely continuous probability measure $\mu$ on the measurable space $\left([0,1], \mathcal{B}_{[0,1]}\right)$ such that $\mathbf{S}(\widetilde{U})=\mathbf{S}^{\mu}(\widetilde{U})$ for all $\widetilde{U} \in$ $\mathcal{F}_{c}^{*}\left(\mathbb{R}^{p}\right)$ (see, for instance, Vetterlein and Navara [34] for a, rather cumbersome, construction of $\mu$ ).

On the basis of these two components (namely, the support function and the Steiner point) we obtain the following representation of fuzzy vectors.

Definition 2.1. Given an absolutely continuous probability measure $\mu$ on the measurable space $\left([0,1], \mathcal{B}_{[0,1]}\right)$, the $\mu$-support/Steiner representation of $\widetilde{U} \in \mathcal{F}_{c}^{*}\left(\mathbb{R}^{p}\right)$ is given by the vectorial value

$$
\begin{gathered}
\boldsymbol{v}_{\widetilde{U}}^{\mu}:[0,1] \times \mathbb{S}^{p-1} \rightarrow \mathbb{R}^{p} \times \mathbb{R} \\
(\alpha, \mathbf{u}) \mapsto \boldsymbol{v}_{\widetilde{U}}^{\mu}(\alpha, \mathbf{u})=\left(\mathbf{S}^{\mu}(\widetilde{U}), s_{\widetilde{U}}(\alpha, \mathbf{u})\right) .
\end{gathered}
$$

For the given measure $\mu$, the $\boldsymbol{v}^{\mu}$ function can be stated as the function

$$
\boldsymbol{v}^{\mu}: \mathcal{F}_{c}^{*}\left(\mathbb{R}^{p}\right) \rightarrow \mathbb{R}^{p} \times \mathbb{H}_{2}, \quad \boldsymbol{v}^{\mu}(\widetilde{U}) \mapsto \boldsymbol{v}_{\widetilde{U}}^{\mu}=\left(\mathbf{S}^{\mu}(\widetilde{U}), s_{\widetilde{U}}\right)
$$


where $\mathbb{H}_{2}=\left\{L^{2}\right.$ type real-valued functions defined on $[0,1] \times \mathbb{S}^{p-1}$ w.r.t. $\left.\lambda_{p}, \ell\right\}$ (with $\ell=$ Lebesgue measure on $\left([0,1], \mathcal{B}_{[0,1]}\right)$ ).

As an immediate consequence from the already mentioned support function characterization of a fuzzy vector (see Butnariu et al. [6]) and the definition of the Steiner point, the following result establishes necessary and sufficient conditions to characterize each fuzzy vector by a support/Steiner representation. Thus,

Proposition 2.1. If there exist a function $s:[0,1] \times \mathbb{S}^{p-1} \rightarrow \mathbb{R}$ fulfilling conditions s.i) and s.ii), and an absolutely continuous probability measure $\mu$ on $\left([0,1], \mathcal{B}_{[0,1]}\right)$, then there exists a unique fuzzy vector $\widetilde{U} \in \mathcal{F}_{c}^{*}\left(\mathbb{R}^{p}\right)$ such that

$$
\boldsymbol{v}^{\mu}(\widetilde{U})=(\mathbf{m}, s)
$$

with $\mathbf{m}=\iint_{[0,1] \times \mathbb{S}^{p-1}} \mathbf{u} \cdot s(\alpha, \mathbf{u}) d \lambda_{p}(\mathbf{u}) d \mu(\alpha)$.

More generally, and based on Vetterlein and Navara's results [34, 35], we can trivially ensure that

Proposition 2.2. Given an $\mathbb{R}^{p} \times \mathbb{H}_{2}$-valued function $\boldsymbol{v}: \mathcal{F}_{c}^{*}\left(\mathbb{R}^{p}\right) \rightarrow \mathbb{R}^{p} \times \mathbb{H}_{2}$ such that $\boldsymbol{v}=(\mathbf{S}, h)$, where

- $h: \mathcal{F}_{c}^{*}\left(\mathbb{R}^{p}\right) \rightarrow \mathbb{H}_{2}$ is such that for each $\widetilde{U} \in \mathcal{F}_{c}^{*}\left(\mathbb{R}^{p}\right)$ the real-valued function $h(\widetilde{U}):[0,1] \times \mathbb{S}^{p-1} \rightarrow \mathbb{R}$ satisfies conditions s.i) and s.ii),

- and $\mathbf{S}: \mathcal{F}_{c}^{*}\left(\mathbb{R}^{p}\right) \rightarrow \mathbb{R}^{p}$ is a vectorial-valued function satisfying conditions $\mathbf{S} . i)-\mathbf{S} . i v)$,

there exists an absolutely continuous probability measure $\mu$ on the measurable space $\left([0,1], \mathcal{B}_{[0,1]}\right)$ such that $\boldsymbol{v}=\boldsymbol{v}^{\mu}$.

Remark 2.1. The last two propositions indicate that, in contrast to the support function representation for fuzzy vectors (characterizing them in terms of their 'boundaries'), the support/Steiner representation of fuzzy vectors characterize them through their 'shape' and 'central location'. Furthermore, sufficient conditions can be given to determine a fuzzy vector what, as already pointed out, is an essential difference with the so-called mid-point/spread representation for fuzzy vectors (see Trutschnig et al. [33] with mid $s_{\widetilde{U}}(\alpha, \mathbf{u})$ $=\left[s_{\widetilde{U}}(\alpha, \mathbf{u})-s_{\widetilde{U}}(\alpha,-\mathbf{u})\right] / 2$ and $\left.\operatorname{spr} s_{\widetilde{U}}(\alpha, \mathbf{u})=\left[s_{\widetilde{U}}(\alpha,-\mathbf{u})+s_{\widetilde{U}}(\alpha, \mathbf{u})\right] / 2\right)$ : there do not exist sufficient conditions for the mid-point/spread representation to characterize fuzzy vectors (see a detailed discussion about the onedimensional case in Sinova et al. [28]). 


\section{An $L^{2}$ generalized metric between fuzzy vectors based on their support functions and generalized Steiner points}

In a recent paper (Sinova et al. [28]), an $L^{2}$ metric between fuzzy numbers has been introduced. This metric is based on a representation for which there exist sufficient conditions characterizing fuzzy numbers. This representation takes into account separately a 'central location' indicator of the fuzzy number and, level-wise and as indicators of their 'shape', the left and right deviations of the extreme points w.r.t. the central location.

This metric can be extended to deal with fuzzy vectors by using their (Puri and Ralescu) support function, for which sufficient conditions to characterize them are well-known (see, for instance, Puri and Ralescu [24], Diamond and Kloeden [12], Liang et al. [21]). The metric considers

- as indicator of the central location of a fuzzy vector, the $\mu$-support function-based Steiner point;

- and, level-wise, and as an indicator of its shape, the vectorial-valued deviation w.r.t. the central location.

The family of metrics is now introduced as follows:

Definition 3.1. Given an absolutely continuous probability measure $\varphi$ on $\left([0,1], \mathcal{B}_{[0,1]}\right)$ with positive mass function on $(0,1)$, and a parameter $\theta \in$ $(0,1]$, the support/Steiner-based $L^{2}$ metric is the mapping $\mathfrak{D}_{\theta}^{\varphi}: \mathcal{F}_{c}^{*}\left(\mathbb{R}^{p}\right)$ $\times \mathcal{F}_{c}^{*}\left(\mathbb{R}^{p}\right) \rightarrow[0,+\infty)$ such that for $\widetilde{U}, \widetilde{V} \in \mathcal{F}_{c}^{*}\left(\mathbb{R}^{p}\right)$

$$
\begin{gathered}
\mathfrak{D}_{\theta}^{\varphi}(\widetilde{U}, \widetilde{V})=\left[(1-\theta)\left\|\mathbf{S}^{\varphi}(\widetilde{U})-\mathbf{S}^{\varphi}(\widetilde{V})\right\|^{2}\right. \\
\left.+\theta \iint_{[0,1] \times \mathbb{S}^{p-1}}\left[s_{\widetilde{U}}(\alpha, \mathbf{u})-s_{\widetilde{V}}(\alpha, \mathbf{u})\right]^{2} d \lambda_{p}(\mathbf{u}) d \varphi(\alpha)\right]^{1 / 2}=\left[\left\|\mathbf{S}^{\varphi}(\widetilde{U})-\mathbf{S}^{\varphi}(\widetilde{V})\right\|^{2}\right. \\
\left.+\theta \iint_{[0,1] \times \mathbb{S}^{p-1}}\left\|\operatorname{dev}_{\widetilde{U}}^{\varphi}(\alpha, \mathbf{u})-\operatorname{dev}_{\widetilde{V}}^{\varphi}(\alpha, \mathbf{u})\right\|^{2} d \lambda_{p}(\mathbf{u}) d \varphi(\alpha)\right]^{1 / 2}
\end{gathered}
$$

where $\operatorname{dev}_{\widetilde{U}}^{\varphi}(\alpha, \mathbf{u})=\mathbf{u} \cdot s_{\widetilde{U}}(\alpha, \mathbf{u})-\mathbf{S}^{\varphi}(\widetilde{U})$.

The mapping $\mathfrak{D}_{\theta}^{\varphi}$ can be also expressed in terms of the well-known extended distance between fuzzy vectors by Diamond and Körner [13], which is given by

$$
\rho_{2}^{\varphi}(\widetilde{U}, \widetilde{V})=\sqrt{\iint_{[0,1] \times \mathbb{S}^{p-1}}\left[s_{\widetilde{U}}(\alpha, \mathbf{u})-s_{\widetilde{V}}(\alpha, \mathbf{u})\right]^{2} d \lambda_{p}(\mathbf{u}) d \varphi(\alpha)} .
$$


More concretely,

$$
\mathfrak{D}_{\theta}^{\varphi}(\widetilde{U}, \widetilde{V})=\sqrt{(1-\theta)\left\|\mathbf{S}^{\varphi}(\widetilde{U})-\mathbf{S}^{\varphi}(\tilde{V})\right\|^{2}+\theta\left[\rho_{2}^{\varphi}(\widetilde{U}, \widetilde{V})\right]^{2}} .
$$

The mapping $\mathfrak{D}_{\theta}^{\varphi}$ is a distance between fuzzy vectors. Thus,

Proposition 3.1. $\left(\mathcal{F}_{c}^{*}(\mathbb{R}), \mathfrak{D}_{\theta}^{\varphi}\right)$ is a metric space.

Proof. Indeed, since the square of $\mathfrak{D}_{\theta}^{\varphi}$ is the sum of a semi-metric plus the square of a metric defined on $\mathcal{F}_{c}^{*}(\mathbb{R})$, it is trivially nonnegative (i.e., it satisfies the separation axiom), it fulfils the identity of indiscernibles (or coincidence axiom), and it is symmetric. Furthermore, if $\widetilde{U}, \widetilde{V}, \widetilde{W} \in \mathcal{F}_{c}^{*}\left(\mathbb{R}^{p}\right)$, then since $\|\cdot\|$ is a norm and $\rho_{2}^{\varphi}$ is a metric,

$$
\begin{gathered}
{\left[\mathfrak{D}_{\theta}^{\varphi}(\widetilde{U}, \widetilde{V})\right]^{2} \leq(1-\theta)\left[\left\|\mathbf{S}^{\varphi}(\widetilde{U})-\mathbf{S}^{\varphi}(\widetilde{W})\right\|+\left\|\mathbf{S}^{\varphi}(\widetilde{W})-\mathbf{S}^{\varphi}(\widetilde{V})\right\|\right]^{2}} \\
+\theta\left[\rho_{2}^{\varphi}(\widetilde{U}, \widetilde{W})+\rho_{2}^{\varphi}(\widetilde{W}, \widetilde{V})\right]^{2}=\left[\mathfrak{D}_{\theta}^{\varphi}(\widetilde{U}, \widetilde{W})\right]^{2}+\left[\mathfrak{D}_{\theta}^{\varphi}(\widetilde{W}, \widetilde{V})\right]^{2} \\
+2(1-\theta)\left\|\mathbf{S}^{\varphi}(\widetilde{U})-\mathbf{S}^{\varphi}(\widetilde{W})\right\| \cdot\left\|\mathbf{S}^{\varphi}(\widetilde{W})-\mathbf{S}^{\varphi}(\widetilde{V})\right\|+2 \theta \rho_{2}^{\varphi}(\widetilde{U}, \widetilde{W}) \cdot \rho_{2}^{\varphi}(\widetilde{W}, \widetilde{V}),
\end{gathered}
$$

and, due to the fact that

$$
\begin{gathered}
{\left[(1-\theta)\left\|\mathbf{S}^{\varphi}(\widetilde{U})-\mathbf{S}^{\varphi}(\widetilde{W})\right\| \cdot\left\|\mathbf{S}^{\varphi}(\widetilde{W})-\mathbf{S}^{\varphi}(\widetilde{V})\right\|+\theta \rho_{2}^{\varphi}(\widetilde{U}, \widetilde{W}) \cdot \rho_{2}^{\varphi}(\widetilde{W}, \widetilde{V})\right]^{2}} \\
=\left[\mathfrak{D}_{\theta}^{\varphi}(\widetilde{U}, \widetilde{W})\right]^{2} \cdot\left[\mathfrak{D}_{\theta}^{\varphi}(\widetilde{W}, \widetilde{V})\right]^{2} \\
-\theta(1-\theta)\left[\left\|\mathbf{S}^{\varphi}(\widetilde{U})-\mathbf{S}^{\varphi}(\widetilde{W})\right\| \cdot \rho_{2}^{\varphi}(\widetilde{W}, \widetilde{V})-\left\|\mathbf{S}^{\varphi}(\widetilde{W})-\mathbf{S}^{\varphi}(\widetilde{V})\right\| \cdot \rho_{2}^{\varphi}(\widetilde{U}, \widetilde{W})\right]^{2} \\
\leq\left[\mathfrak{D}_{\theta}^{\varphi}(\widetilde{U}, \widetilde{W})\right]^{2} \cdot\left[\mathfrak{D}_{\theta}^{\varphi}(\widetilde{W}, \widetilde{V})\right]^{2},
\end{gathered}
$$

we have that

$$
\left[\mathfrak{D}_{\theta}^{\varphi}(\widetilde{U}, \widetilde{V})\right]^{2} \leq\left[\mathfrak{D}_{\theta}^{\varphi}(\widetilde{U}, \widetilde{W})+\mathfrak{D}_{\theta}^{\varphi}(\widetilde{W}, \widetilde{V})\right]^{2}
$$

so that $\mathfrak{D}_{\theta}^{\varphi}$ is subadditive, that is, it satisfies the triangle inequality.

The metric $\mathfrak{D}_{\theta}^{\varphi}$ is an extension to the $p$-dimensional case of the wabl/ldev/rdev $L^{2}$ metric defined by Sinova et al. [28] for the one-dimensional case as follows

$$
\mathfrak{D}_{\theta}^{\varphi}(\widetilde{U}, \widetilde{V})=\left[\left(\mathrm{wabl}^{\varphi} \widetilde{U}-\mathrm{wabl}^{\varphi} \widetilde{V}\right)^{2}\right.
$$




$$
\left.+\theta \int_{[0,1]}\left(\frac{1}{2}\left[\operatorname{ldev}_{\widetilde{U}}^{\varphi}(\alpha)-\operatorname{ldev}_{\widetilde{V}}^{\varphi}(\alpha)\right]^{2}+\frac{1}{2}\left[\operatorname{rdev}_{\widetilde{U}}^{\varphi}(\alpha)-\operatorname{rdev}_{\widetilde{V}}^{\varphi}(\alpha)\right]^{2}\right) d \varphi(\alpha)\right]^{1 / 2}
$$

where the weighted averaging based on levels (see Yager [36], Campos and González [9], Nasibov [22]) is given by

$$
\operatorname{wabl}^{\varphi} \widetilde{U}=\int_{[0,1]} \operatorname{mid} \widetilde{U}_{\alpha} d \varphi(\alpha)=\mathbf{S}^{\varphi}(\widetilde{U})
$$

with mid $\widetilde{U}_{\alpha}=$ centre of $\widetilde{U}_{\alpha}=\left[\inf \widetilde{U}_{\alpha}+\sup \widetilde{U}_{\alpha}\right] / 2$,

$$
\begin{gathered}
\operatorname{ldev}_{\widetilde{U}}^{\varphi}(\alpha)=\operatorname{wabl}^{\varphi} \widetilde{U}-\inf \widetilde{U}_{\alpha}=-\operatorname{dev}_{\widetilde{U}}^{\varphi}(\alpha,-1), \\
\operatorname{rdev}_{\widetilde{U}}^{\varphi}(\alpha)=\sup \widetilde{U}_{\alpha}-\operatorname{wabl}^{\varphi} \widetilde{U}=\operatorname{dev}_{\widetilde{U}}^{\varphi}(\alpha, 1) .
\end{gathered}
$$

This is due to the fact that $\mathbb{S}^{0}=\{-1,1\}, s_{\widetilde{U}}(\alpha, 1)=\sup \widetilde{U}_{\alpha}, s_{\widetilde{U}}(\alpha,-1)$ $=-\inf \widetilde{U}_{\alpha}$, and $\lambda_{1}(0)=\lambda_{1}(1)=1 / 2$.

Both, the parameter $\theta$ and the measure $\varphi$, do not have a stochastic meaning. Thus, parameter $\theta$ in the distance weighs the influence of the 'deviation in shape' between the fuzzy vectors (quantified through $\mathbf{d e v}^{\varphi}$ ) with respect to the influence of their 'deviation in central location' (quantified through the generalized Steiner point $\mathbf{S}^{\varphi}$ ). The choice of $\varphi$ allows us to weigh the influence of each $\alpha$-level (i.e., the different degrees of 'compatibility').

The support/Steiner representation of fuzzy vectors allows us to induce a parametric family of $L^{2}$ metrics on $\mathcal{F}_{c}^{*}\left(\mathbb{R}^{p}\right)$. For a given weighting measure $\varphi$, and for any $f \in \mathbb{H}_{2}, \alpha \in[0,1]$ and $u \in \mathbb{S}^{p-1}$ define

$$
\mathbf{S}^{\varphi}(f)=\iint_{[0,1] \times \mathbb{S}^{p-1}} \mathbf{u} \cdot f(\alpha, \mathbf{u}) d \lambda_{p}(\mathbf{u}) d \varphi(\alpha) .
$$

An inner product in $\mathbb{R}^{p} \times \mathbb{H}_{2}$ can be defined as follows: let $\theta \in(0,1]$, and let $\varphi$ be a weighting measure formalized as an absolutely continuous probability measure on $\left([0,1], \mathcal{B}_{[0,1]}\right)$ with positive mass function in $(0,1)$. For $\mathbf{x}, \mathbf{y} \in \mathbb{R}^{p}$ and $f, g \in \mathbb{H}_{2}$, consider the inner product

$$
\langle(\mathbf{x}, f),(\mathbf{y}, g)\rangle_{\theta}^{\varphi}=\int_{(0,1]} \int_{\mathbb{S}^{p-1}}\langle(\mathbf{x}, f(\alpha, \mathbf{u})),(\mathbf{y}, g(\alpha, \mathbf{u}))\rangle_{\theta} d \lambda_{p}(\mathbf{u}) d \varphi(\alpha),
$$

where the Euclidean inner product $\langle\cdot, \cdot\rangle_{\theta}$ on $\mathbb{R}^{p} \times \mathbb{R}$ is based on the weighted dot product given by

$$
\left\langle\left(\mathbf{x}_{1}, x_{2}\right),\left(\mathbf{y}_{1}, y_{2}\right)\right\rangle_{\theta}=(1-\theta) \mathbf{x}_{1} \cdot \mathbf{y}_{1}+\theta x_{2} y_{2} .
$$

Then, the following results hold: 
Proposition 3.2. Let $\varphi$ be an arbitrarily fixed absolutely continuous probability measure on $\left([0,1], \mathcal{B}_{[0,1]}\right)$, with positive mass function on $(0,1)$, and $\theta \in(0,1]$ be a weighting parameter. Then,

i) $\left(\mathbb{R}^{p} \times \mathbb{H}_{2},\langle\cdot, \cdot\rangle_{\theta}^{\varphi}\right)$ is a Hilbert space.

ii) $\mathfrak{D}_{\theta}^{\varphi}$ is an $L^{2}$-type metric, and it is translational invariant and invariant under opposition (multiplication by -1 ).

iii) For fixed $\varphi$, the $\boldsymbol{v}^{\varphi}$ function satisfies that

- $\boldsymbol{v}^{\varphi}$ is an isometry from $\left(\mathcal{F}_{c}^{*}\left(\mathbb{R}^{p}\right), \mathfrak{D}_{\theta}^{\varphi}\right)$ into $\left(\mathbb{R}^{p} \times \mathbb{H}_{2},\langle\cdot, \cdot\rangle_{\theta}^{\varphi}\right)$,

- $\boldsymbol{v}^{\varphi}(\widetilde{U}+\widetilde{V})=\boldsymbol{v}^{\varphi}(\widetilde{U})+\boldsymbol{v}^{\varphi}(\widetilde{V})$ for all $\widetilde{U}, \widetilde{V} \in \mathcal{F}_{c}^{*}\left(\mathbb{R}^{p}\right)$,

- $\boldsymbol{v}^{\varphi}(\gamma \cdot \widetilde{U})=\gamma \cdot \boldsymbol{v}^{\varphi}(\widetilde{U})$ for all $\widetilde{U} \in \mathcal{F}_{c}^{*}\left(\mathbb{R}^{p}\right)$ and $\gamma>0$;

Consequently, the $\boldsymbol{v}^{\varphi}$ function preserves the semilinearity of $\mathcal{F}_{c}^{*}\left(\mathbb{R}^{p}\right)$ and relates the fuzzy arithmetic to the vectorial-valued functional arithmetic, which implies that $\mathcal{F}_{c}^{*}\left(\mathbb{R}^{p}\right)$ can be isometrically embedded into a convex cone of the Hilbert space $\left(\mathbb{R}^{p} \times \mathbb{H}_{2},\langle\cdot, \cdot\rangle_{\theta}^{\varphi}\right)$.

Proof. Indeed,

i) $\langle\cdot, \cdot\rangle_{\theta}^{\varphi}$ is an inner product on $\mathbb{R}^{p} \times \mathbb{H}_{2}$ because of the way it has been constructed (see details above). Furthermore, it will be proved now that $\left(\mathbb{R}^{p} \times \mathbb{H}_{2}, \mathfrak{D}_{\theta}^{\varphi}(\cdot, \cdot)\right)$, where

$$
\mathfrak{D}_{\theta}^{\varphi}((\mathbf{x}, f),(\mathbf{y}, g))=\left[(1-\theta)\|\mathbf{x}-\mathbf{y}\|^{2}+\theta\left(\rho_{2}^{\varphi}(f, g)\right)^{2}\right]^{1 / 2},
$$

is a complete metric space. For fixed $\theta \in(0,1)$ and $\varphi$, if $\left\{\left(\mathbf{x}_{n}, f_{n}\right)\right\}_{n}$ is a Cauchy sequence on $\mathbb{R}^{p} \times \mathbb{H}_{2}$, that is, for all $\varepsilon>0$ there exists $N_{\varepsilon} \in \mathbb{N}$ such that for all $m, n>N_{\varepsilon}$

$$
\mathfrak{D}_{\theta}^{\varphi}\left(\left(\mathbf{x}_{m}, f_{m}\right),\left(\mathbf{x}_{n}, f_{n}\right)\right)<\varepsilon,
$$

then, let us see that $\left\{\left(x_{n}, f_{n}\right)\right\}_{n}$ converges. For all $\varepsilon^{*}>0$, define $\varepsilon=\min \{\sqrt{1-\theta}, \sqrt{\theta}\} \cdot \varepsilon^{*}>0$. Using the property above, there exists $N_{\varepsilon^{*}}=N_{\varepsilon} \in \mathbb{N}$ such that for all $m, n>N_{\varepsilon^{*}}$,

$-\sqrt{1-\theta} \cdot\left\|\mathbf{x}_{m}-\mathbf{x}_{n}\right\|<\varepsilon$, so $\left\|\mathbf{x}_{m}-\mathbf{x}_{n}\right\|<\varepsilon^{*}$, whence because of $\left(\mathbb{R}^{p},\|\cdot\|\right)$ being a Hilbert space there exists $\mathbf{x} \in \mathbb{R}^{p}$ such that $\lim _{n \rightarrow \infty}\left\|\mathbf{x}_{n}-\mathbf{x}\right\|=0$;

$-\sqrt{\theta} \cdot \rho_{2}^{\varphi}\left(f_{m}, f_{n}\right)<\varepsilon$, so $\rho_{2}^{\varphi}\left(f_{m}, f_{n}\right)<\varepsilon^{*}$, whence because of $\left(\mathbb{H}_{2}, \rho_{2}^{\varphi}\right)$ being a Hilbert space there exists $f \in \mathbb{H}_{2}$ such that $\lim _{n \rightarrow \infty} \rho_{2}^{\varphi}\left(f_{n}\right.$, $f)=0$. 
Thus, there exists $(\mathbf{x}, f) \in \mathbb{R}^{p} \times \mathbb{H}_{2}$ such that

$$
\lim _{n \rightarrow \infty} \mathfrak{D}_{\theta}^{\varphi}\left(\left(\mathbf{x}_{n}, f_{n}\right),(\mathbf{x}, f)\right)=0
$$

which proves the completeness of the considered metric space.

ii) The proof for this assertion is straightforward, because of the wellknown properties for $\|\cdot\|$ and $\rho_{2}^{\varphi}$.

iii) Obviously, if $\|\cdot\|_{\theta}^{\varphi}$ denotes the norm associated with the inner product $\langle\cdot, \cdot\rangle_{\theta}^{\varphi}$, we have that

$$
\begin{gathered}
\mathfrak{D}_{\theta}^{\varphi}(\widetilde{U}, \widetilde{V})=\left\|\boldsymbol{v}^{\varphi}(\widetilde{U})-\boldsymbol{v}^{\varphi}(\widetilde{V})\right\|_{\theta}^{\varphi} \\
=\left[\left\langle\boldsymbol{v}^{\varphi}(\widetilde{U})-\boldsymbol{v}^{\varphi}(\widetilde{V}), \boldsymbol{v}^{\varphi}(\widetilde{U})-\boldsymbol{v}^{\varphi}(\widetilde{V})\right\rangle_{\theta}^{\varphi}\right]^{1 / 2},
\end{gathered}
$$

so that $\boldsymbol{v}^{\varphi}$ is an isometry.

Moreover, due to the properties of the generalized Steiner points and the support functions, the equivariance of $\boldsymbol{v}^{\varphi}$ w.r.t. the sum and the multiplication times positive scalars, as well as the preservation of the semilinearity, are immediately concluded.

The metric space $\left(\mathcal{F}_{c}^{*}\left(\mathbb{R}^{p}\right), \mathfrak{D}_{\theta}^{\varphi}\right)$ is separable. This assertion is justified by the fact that $\mathfrak{D}_{\theta}^{\varphi}$ is topologically equivalent (in fact, strongly equivalent) to the metric $\rho_{2}^{\varphi}$, as we will see in the next proposition.

Proposition 3.3. Let $\theta \in(0,1]$ be a weight parameter, and let $\varphi$ be an arbitrarily fixed absolutely continuous probability measure on $\left([0,1], \mathcal{B}_{[0,1]}\right)$ with positive mass function in $(0,1)$. The metric $\mathfrak{D}_{\theta}^{\varphi}$ is topologically equivalent to the metric $\rho_{2}^{\varphi}$ on $\mathcal{F}_{c}^{*}\left(\mathbb{R}^{p}\right)$. More precisely,

$$
\sqrt{\theta} \cdot \rho_{2}^{\varphi}(\widetilde{U}, \widetilde{V}) \leq \mathfrak{D}_{\theta}^{\varphi}(\widetilde{U}, \widetilde{V}) \leq \rho_{2}^{\varphi}(\widetilde{U}, \widetilde{V})
$$

for all $\widetilde{U}, \widetilde{V} \in \mathcal{F}_{c}^{*}\left(\mathbb{R}^{p}\right)$.

Proof. Indeed,

$$
\begin{aligned}
\mathfrak{D}_{\theta}^{\varphi}(\widetilde{U}, \widetilde{V}) & =\sqrt{(1-\theta)\left\|\mathbf{S}^{\varphi}(\widetilde{U})-\mathbf{S}^{\varphi}(\widetilde{V})\right\|^{2}+\theta\left[\rho_{2}^{\varphi}(\widetilde{U}, \widetilde{V})\right]^{2}} \\
& \geq \sqrt{\theta\left[\rho_{2}^{\varphi}(\widetilde{U}, \widetilde{V})\right]^{2}}=\sqrt{\theta} \cdot \rho_{2}^{\varphi}(\widetilde{U}, \widetilde{V}) .
\end{aligned}
$$


On the other hand,

$$
\begin{gathered}
\left\|\mathbf{S}^{\varphi}(\widetilde{U})-\mathbf{S}^{\varphi}(\widetilde{V})\right\|^{2}=\left\|\iint_{[0,1] \times \mathbb{S}^{p-1}} \mathbf{u} \cdot\left[s_{\widetilde{U}}(\alpha, \mathbf{u})-s_{\widetilde{V}}(\alpha, \mathbf{u})\right] d \lambda_{p}(\mathbf{u}) d \varphi(\alpha)\right\|^{2} \\
\leq \iint_{[0,1] \times \mathbb{S}^{p-1}}\left\|\mathbf{u} \cdot\left[s_{\widetilde{U}}(\alpha, \mathbf{u})-s_{\widetilde{V}}(\alpha, \mathbf{u})\right]\right\|^{2} d \lambda_{p}(\mathbf{u}) d \varphi(\alpha) \\
=\iint_{[0,1] \times \mathbb{S}^{p-1}}\left\|s_{\widetilde{U}}(\alpha, \mathbf{u})-s_{\widetilde{V}}(\alpha, \mathbf{u})\right\|^{2} d \lambda_{p}(\mathbf{u}) d \varphi(\alpha)=\left[\rho_{2}^{\varphi}(\widetilde{U}, \widetilde{V})\right]^{2}
\end{gathered}
$$

whence

$$
\mathfrak{D}_{\theta}^{\varphi}(\widetilde{U}, \widetilde{V}) \leq \rho_{2}^{\varphi}(\widetilde{U}, \widetilde{V})
$$

As a consequence from this result, $\mathfrak{D}_{\theta}^{\varphi}$ induces on $\mathcal{F}_{c}^{*}\left(\mathbb{R}^{p}\right)$ the same topology as

$$
d_{2}^{\varphi}(\widetilde{U}, \widetilde{V})=\sqrt{\int_{[0,1]} d_{H}\left(\widetilde{U}_{\alpha}, \widetilde{V}_{\alpha}\right)^{2} d \varphi(\alpha)},
$$

where $d_{H}$ denotes the well-known Hausdorff metric on the space of nonempty compact subsets of $\mathbb{R}^{p}$. Furthermore, $\left(\mathcal{F}_{c}^{*}\left(\mathbb{R}^{p}\right), d_{2}^{\varphi}\right)$ is a separable metric space. The proofs for the last two assertions can be straightforwardly derived by simply adapting the ones by Diamond and Kloeden [10] (see the comments after Theorem 2 and the proof of Theorem 3, pp. 244-245) replacing $d \ell(\alpha)$ (or, equivalently, $d \alpha$ ) by $d \varphi(\alpha)$. In the same way, one can ensure that $\mathfrak{D}_{\theta}^{\varphi}$ induces on $\mathcal{F}_{c}^{*}\left(\mathbb{R}^{p}\right)$ the same topology as the metric $D_{\theta}^{\varphi}$ by Trutschnig et al. [33].

The practical computation of $\mathfrak{D}_{\theta}^{\varphi}$ in the case $p=1$ is quite simple and easy to implement in any programming language. Nevertheless, when $p>1$ the situation usually becomes much more complex. This is a consequence of the computational difficulties involved in determining most of the support functions of fuzzy vectors in practice (see Ghosh and Kumar [17] for some details about the two-dimensional set-valued case).

As an example illustrating the use of $\mathfrak{D}_{\theta}^{\varphi}$ in one of the simplest fuzzy vector-valued situations, we are now going to compute the distance of two conical fuzzy vectors arisen in an approach to the tone and color triangle designs (see Sugano [29, 30, 31] and Sugano et al. [32]). 
Example 3.1. Sugano and collaborators have developed studies on a system of the three primary colors RGB presented on a color triangle. The usual triangle involves sixty-six fuzzy inputs on parts of the tone triangle designated as darkness-blackness, lightness-whiteness and chromaticness. The fuzzy inputs are formed by right circular conical fuzzy vectors, and they can mutually overlap (actually, any fuzzy input overlaps with some other ones). The two considered (horizontal) dimensions correspond to whiteness and chromaticness, and the extreme labels are No. 1: black, No. 11: white, and No. 66: red (maximum chromaticness). Of course, one can also consider around each of the sixty-six inputs many neighbouring colors.

Figure 1 displays the general scheme but only three fuzzy inputs have been represented, namely:

- No. 1 , corresponding to the right cone $\widetilde{C}^{1}$ such that for each $\alpha \in[0,1]$

$$
\widetilde{C}_{\alpha}^{1}=\text { circle with centre }(0,0) \text { and radius } 10(1-\alpha),
$$

- No. 36, corresponding to the right cone $\widetilde{C}^{36}$ such that for each $\alpha \in[0,1]$

$$
\widetilde{C}_{\alpha}^{36}=\text { circle with centre }(15 \sqrt{3}, 65) \text { and radius } 10(1-\alpha),
$$

- No. 58, corresponding to the right cone $\widetilde{C}^{58}$ such that for each $\alpha \in[0,1]$

$$
\widetilde{C}_{\alpha}^{58}=\text { circle with centre }(35 \sqrt{3}, 45) \text { and radius } 10(1-\alpha) .
$$

For the rest of the sixty-six main color inputs, only their 0.5-levels have been graphically displayed.

The support function associated with a right circular conical fuzzy vector $\widetilde{C}$ such that, for each $\alpha \in[0,1]$, the $\alpha$-level corresponds to $\widetilde{C}_{\alpha}=$ circle with centre $(x, y)$ and radius $r(1-\alpha)$, is given for $\mathbf{u}=\left(\cos \beta_{\mathbf{u}}, \sin \beta_{\mathbf{u}}\right) \in \mathbb{S}^{1}$ $=$ circumference with centre $(0,0)$ and radius 1 by

$$
s_{\widetilde{C}}(\alpha, \mathbf{u})=x \cdot \cos \beta_{\mathbf{u}}+y \cdot \sin \beta_{\mathbf{u}}+r(1-\alpha) .
$$

Consequently, one can obtain the following distances (which in this case, and because of the 'shape' of the $\alpha$-levels of the three conical fuzzy vectors coinciding, they are irrespective of the chosen $\varphi$ )

$$
\left[\mathfrak{D}_{\theta}^{\varphi}\left(\widetilde{C}^{1}, \widetilde{C}^{36}\right)\right]^{2}=(1-\theta)\|(0,0)-(15 \sqrt{3}, 65)\|^{2}
$$




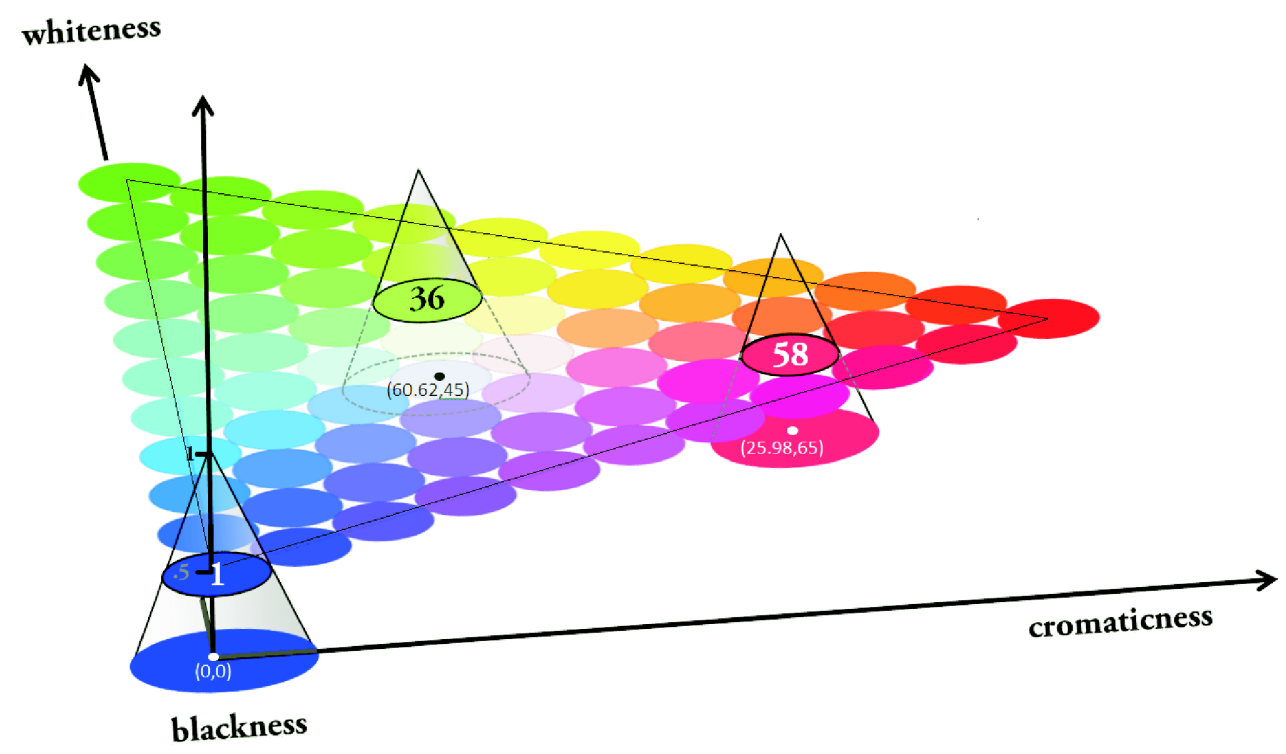

Figure 1: Conical fuzzy vectors in the usual color triangle

$$
\begin{gathered}
+\frac{\theta}{2 \pi} \iint_{[0,1] \times[0,2 \pi)}[10(1-\alpha)-15 \sqrt{3} \cos \beta-65 \sin \beta-10(1-\alpha)]^{2} d \beta d \varphi(\alpha) \\
=4900(1-\theta), \\
{\left[\mathfrak{D}_{\theta}^{\varphi}\left(\widetilde{C}^{1}, \widetilde{C}^{58}\right)\right]^{2}=(1-\theta)\|(0,0)-(35 \sqrt{3}, 45)\|^{2}} \\
+\frac{\theta}{2 \pi} \iint_{[0,1] \times[0,2 \pi)}[10(1-\alpha)-35 \sqrt{3} \cos \beta-45 \sin \beta-10(1-\alpha)]^{2} d \beta d \varphi(\alpha) \\
\quad=5700(1-\theta), \\
{\left[\mathfrak{D}_{\theta}^{\varphi}\left(\widetilde{C}^{36}, \widetilde{C}^{58}\right)\right]^{2}=(1-\theta)\|(15 \sqrt{3}, 65)-(35 \sqrt{3}, 45)\|^{2}} \\
+\frac{\theta}{2 \pi} \iint_{[0,1] \times[0,2 \pi)}[15 \sqrt{3} \cos \beta+65 \sin \beta+10(1-\alpha) \\
-35 \sqrt{3} \cos \beta-45 \sin \beta-10(1-\alpha)]^{2} d \beta d \varphi(\alpha)=1600(1-\theta) .
\end{gathered}
$$

Therefore, and whatever the weight $\theta$ may be, we can conclude that the fuzzy inputs No. 36 and No. 58 are closer than No. 1 and No. 36 and than No. 1 and No. 58. 


\section{The mean square error associated with a random fuzzy vector based on the support functions and generalized Steiner points}

In Statistics, distances between data or values represent a valuable tool often in connection with the quantification of some errors, like the error in estimating either a parameter of the population distribution or some relevant values, or in connection with the quantification of the deviation between what is stated by a hypothesis about such a distribution and what is evidenced by the available sample information.

Furthermore, due to the nonlinearity of $\mathcal{F}_{c}^{*}\left(\mathbb{R}^{p}\right)$ when it is endowed with the usual fuzzy arithmetic, a well-defined difference '-' between fuzzy vectors cannot be established so that $\widetilde{U}+(\widetilde{V}-\widetilde{U})=\widetilde{V}$ whatever $\widetilde{U}, \widetilde{V} \in \mathcal{F}_{c}^{*}\left(\mathbb{R}^{p}\right)$ may be. It should be noted that, although Hukuhara's difference satisfy the last equality, there are many fuzzy vectors for which such a difference is not defined.

In the real- and vectorial-valued cases, the squared Euclidean 2-norm of the difference of two values is equivalent to the squared Euclidean distance between them, so one can think about proceeding in accordance with the second way in the fuzzy vector-valued case.

In this section, a measure of the mean square error associated with a fuzzy vector-valued random element is to be introduced and analyzed. This measure is based on the $\mathfrak{D}_{\theta}^{\varphi}$ metric defined in Section 3. To formalize adequately this concept, it is necessary to consider an appropriate model for the corresponding random elements. This is given by random fuzzy vectors (or, more generally, random fuzzy sets, originally coined as fuzzy random variables by Puri and Ralescu [25]).

Definition 4.1. Given a probability space $(\Omega, \mathcal{A}, P)$, a mapping $\mathcal{X}: \Omega \rightarrow$ $\mathcal{F}_{c}^{*}\left(\mathbb{R}^{p}\right)$ is said to be a random fuzzy vector associated with it if, for all $\alpha \in(0,1]$, the $\alpha$-level set-valued mapping

$\mathcal{X}_{\alpha}: \Omega \rightarrow \mathcal{K}_{c}\left(\mathbb{R}^{p}\right)=\left\{\right.$ nonempty compact convex sets of $\left.\mathbb{R}^{p}\right\}, \omega \mapsto(\mathcal{X}(\omega))_{\alpha}$,

is a compact convex random set (that is, $\mathcal{X}_{\alpha}$ is a Borel measurable mapping w.r.t. $\mathcal{A}$ and the Borel $\sigma$-field generated by the topology induced by the Hausdorff metric on $\mathcal{K}_{c}\left(\mathbb{R}^{p}\right)$ ). 
In virtue of the fact that $\mathfrak{D}_{\theta}^{\varphi}$ induces the same topology as some other $L^{2}$ metrics on $\mathcal{F}_{c}^{*}\left(\mathbb{R}^{p}\right)$, and on the basis of the results in previous papers (see, for instance, González-Rodríguez et al. [18]), the notion of random fuzzy vector can be equivalently formalized as a Borel-measurable mapping. More concretely,

Definition 4.2. A mapping $\mathcal{X}: \Omega \rightarrow \mathcal{F}_{c}^{*}\left(\mathbb{R}^{p}\right)$ is said to be a random fuzzy vector if $\mathcal{X}$ is a Borel measurable mapping w.r.t. $\mathcal{A}$ and the Borel $\sigma$-field generated by the topology induced by the metric $\mathfrak{D}_{\theta}^{\varphi}$ on $\mathcal{F}_{c}^{*}\left(\mathbb{R}^{p}\right)$.

A crucial implication from the Borel-measurability in Definition 4.2 is that one can properly refer to the distribution induced by a random fuzzy vector and to independent random fuzzy vectors.

A mean square error measure of a fuzzy vector $\widetilde{U}$ as an estimate of the value of a random fuzzy vector $\mathcal{X}$ can be quantified as follows:

Definition 4.3. Given an absolutely continuous probability measure $\varphi$ on $\left([0,1], \mathcal{B}_{[0,1]}\right)$ with positive mass function on $(0,1)$, a parameter $\theta \in(0,1]$, and a random fuzzy vector $\mathcal{X}$, the support/Steiner-based mean square error of the fuzzy vector $\widetilde{U} \in \mathcal{F}_{c}^{*}\left(\mathbb{R}^{p}\right)$ in estimating the value of $\mathcal{X}$ is the real value (or support/Steiner-based mean square error of the random fuzzy vector $\mathcal{X}$ about the fuzzy vector $\widetilde{U}$ ), if it exists, given by the (real-valued) expectation

$$
\mathfrak{m} \mathfrak{s e} e_{\theta}^{\varphi}(\mathcal{X}, \widetilde{U})=E\left(\left[\mathfrak{D}_{\theta}^{\varphi}(\mathcal{X}, \widetilde{U})\right]^{2}\right) .
$$

For an arbitrary random fuzzy vector $\mathcal{X}$, the support/Steiner-based mean square error $\mathfrak{m s e}_{\theta}^{\varphi}(\mathcal{X}, \widetilde{U})$ is minimized at the fuzzy vector $\widetilde{U}=\widetilde{E}(\mathcal{X})$, where $\widetilde{E}(\mathcal{X})$ is the Aumann-type fuzzy mean of $\mathcal{X}$ defined by Puri and Ralescu [25] as follows:

Definition 4.4. Given a probability space $(\Omega, \mathcal{A}, P)$ and an associated random fuzzy vector $\mathcal{X}$ such that $s_{\mathcal{X}} \in L^{1}(\Omega, \mathcal{A}, P)$, the (Aumann-type) mean value of $\mathcal{X}$ is the fuzzy value $\widetilde{E}(\mathcal{X}) \in \mathcal{F}_{c}^{*}\left(\mathbb{R}^{p}\right)$, if it exists, such that for all $\alpha \in(0,1]$

$$
\begin{gathered}
(\widetilde{E}(\mathcal{X}))_{\alpha}=\text { Aumann integral of } \mathcal{X}_{\alpha} \\
=\left\{\int_{\mathbb{R}^{p}} X(\omega) d P(\omega) \text { for all } X: \Omega \rightarrow \mathbb{R}^{p}, X \in L^{1}(\Omega, \mathcal{A}, P), X \in \mathcal{X}_{\alpha} \text { a.s. }[P]\right\} .
\end{gathered}
$$

Equivalently, if $s_{\mathcal{X}} \in L^{1}(\Omega, \mathcal{A}, P)$ then $s_{\widetilde{E}(\mathcal{X})}=E\left(s_{\mathcal{X}}\right)$, with $E$ here denoting the functional-valued expectation of a Hilbert space-valued random element. 
Thus,

Proposition 4.1. Let $\varphi$ be an absolutely continuous probability measure on $\left([0,1], \mathcal{B}_{[0,1]}\right)$ with positive mass function on $(0,1), \theta \in(0,1]$ be a parameter, and $\mathcal{X}$ be a random fuzzy vector associated with the probability space $(\Omega, \mathcal{A}, P)$. If $s_{\mathcal{X}} \in L^{2}(\Omega, \mathcal{A}, P)$ and $\mathbf{u} \cdot s_{\mathcal{X}}(\alpha, \mathbf{u})$ is integrable on $\Omega \times([0,1] \times$ $\left.\mathbb{S}^{p-1}\right)$, then

$$
\widetilde{E}(\mathcal{X})=\arg \min _{\widetilde{U} \in \mathcal{F}_{c}^{*}\left(\mathbb{R}^{p}\right)} \mathfrak{m s e} \mathfrak{s e}_{\theta}^{\varphi}(\mathcal{X}, \widetilde{U})
$$

Proof. Indeed,

$$
\mathfrak{m s e}_{\theta}^{\varphi}(\mathcal{X}, \widetilde{U})=(1-\theta) E\left(\left\|\mathbf{S}^{\varphi}(\mathcal{X})-\mathbf{S}^{\varphi}(\widetilde{U})\right\|^{2}\right)+\theta E\left(\left[\rho_{2}^{\varphi}(\mathcal{X}, \widetilde{U})\right]^{2}\right) .
$$

On one hand, it is well-known that $\widetilde{E}(\mathcal{X})$ is the Fréchet mean associated with $\rho_{2}^{\varphi}$ (and many other $L^{2}$ metrics on $\mathcal{F}_{c}^{*}\left(\mathbb{R}^{p}\right)$ ), that is,

$$
\widetilde{E}(\mathcal{X})=\arg \min _{\widetilde{U} \in \mathcal{F}_{c}^{*}\left(\mathbb{R}^{p}\right)} E\left(\left[\rho_{2}^{\varphi}(\mathcal{X}, \widetilde{U})\right]^{2}\right) .
$$

On the other hand, if $\mathcal{X}$ is a random fuzzy vector, then $\mathbf{S}^{\varphi}(\mathcal{X})$ is a random vector (see, for instance, Aletti and Bongiorno [1], in case $\varphi$ is the Lebesgue measure on $\left([0,1], \mathcal{B}_{[0,1]}\right)$, result that can be straightforwardly extended for any $\varphi$ ). Furthermore, if $\mathbf{E}$ denotes the vectorial-valued expectation of a random vector,

$$
\mathbf{E}\left(\mathbf{S}^{\varphi}(\mathcal{X})\right)=\arg \min _{\widetilde{U} \in \mathcal{F}_{c}^{*}\left(\mathbb{R}^{p}\right)} E\left(\left\|\mathbf{S}^{\varphi}(\mathcal{X})-\mathbf{S}^{\varphi}(\widetilde{U})\right\|^{2}\right)
$$

and, since sufficient conditions allowing us to apply Fubini's Theorem are fulfilled, we have that

$$
\begin{gathered}
\mathbf{E}\left(\mathbf{S}^{\varphi}(\mathcal{X})\right)=\iint_{[0,1] \times \mathbb{S}^{p-1}} \mathbf{E}\left(\mathbf{u} \cdot s_{\mathcal{X}}(\alpha, \mathbf{u})\right) d \lambda_{p}(\mathbf{u}) d \varphi(\alpha) \\
=\iint_{[0,1] \times \mathbb{S}^{p-1}} \mathbf{u} \cdot E\left(s_{\mathcal{X}}(\alpha, \mathbf{u})\right) d \lambda_{p}(\mathbf{u}) d \varphi(\alpha) .
\end{gathered}
$$

Since $E(s \mathcal{X}(\cdot, \cdot))=s_{\widetilde{E}(\mathcal{X})}(\cdot, \cdot)$, then

$$
\mathbf{E}\left(\mathbf{S}^{\varphi}(\mathcal{X})\right)=\iint_{[0,1] \times \mathbb{S}^{p-1}} \mathbf{u} \cdot s_{\widetilde{E}(\mathcal{X})}(\alpha, \mathbf{u}) d \lambda_{p}(\mathbf{u}) d \varphi(\alpha)=\mathbf{S}^{\varphi}(\widetilde{E}(\mathcal{X}))
$$

whence, the result is proved.

Actually, the last result can be also derived from a result extending the decomposition of the mean square error of a random variable. Concretely, 
Proposition 4.2. Let $\varphi$ be an absolutely continuous probability measure on $\left([0,1], \mathcal{B}_{[0,1]}\right)$ with positive mass function on $(0,1), \theta \in(0,1]$ be a parameter, and $\mathcal{X}$ be a random fuzzy vector associated with the probability space $(\Omega, \mathcal{A}, P)$. If $s_{\mathcal{X}} \in L^{2}(\Omega, \mathcal{A}, P)$ and $\mathbf{u} \cdot s_{\mathcal{X}}(\alpha, \mathbf{u})$ is integrable on $\Omega \times([0,1]$ $\left.\times \mathbb{S}^{p-1}\right)$, then

$$
\mathfrak{m} \mathfrak{s e}_{\theta}^{\varphi}(\mathcal{X}, \widetilde{U})=\mathfrak{m} \mathfrak{s e}_{\theta}^{\varphi}(\mathcal{X}, \widetilde{E}(\mathcal{X}))+\left[\mathfrak{D}_{\theta}^{\varphi}(\widetilde{E}(\mathcal{X}), \widetilde{U})\right]^{2} .
$$

Proof. Indeed, under the assumed conditions,

$$
\begin{gathered}
\mathfrak{m s k}_{\theta}^{\varphi}(\mathcal{X}, \widetilde{U})=(1-\theta)\left\{E\left(\left\|\mathbf{S}^{\varphi}(\mathcal{X})-\mathbf{E}\left(\mathbf{S}^{\varphi}(\mathcal{X})\right)\right\|^{2}\right)\right. \\
+E\left(\left\|\mathbf{E}\left(\mathbf{S}^{\varphi}(\mathcal{X})\right)-\mathbf{S}^{\varphi}(\widetilde{U})\right\|^{2}\right) \\
\left.+2\left[\mathbf{E}\left(\mathbf{S}^{\varphi}(\mathcal{X})\right)-\mathbf{S}^{\varphi}(\widetilde{U})\right] \cdot \mathbf{E}\left[\mathbf{S}^{\varphi}(\mathcal{X})-\mathbf{E}\left(\mathbf{S}^{\varphi}(\mathcal{X})\right)\right]\right\} \\
+\theta E\left\{\left[\rho_{2}^{\varphi}(\mathcal{X}, \widetilde{E}(\mathcal{X}))\right]^{2}+\left[\rho_{2}^{\varphi}(\widetilde{E}(\mathcal{X}), \widetilde{U})\right]^{2}\right. \\
\left.+2 \iint_{[0,1] \times \mathbb{S}^{p-1}}\left[s_{\widetilde{E}(\mathcal{X})}(\alpha, \mathbf{u})-s_{\widetilde{U}}(\alpha, \mathbf{u})\right]\left[s \mathcal{X}(\alpha, \mathbf{u})-s_{\widetilde{E}(\mathcal{X})}(\alpha, \mathbf{u})\right] d \lambda_{p}(\mathbf{u}) d \varphi(\alpha)\right\} \\
=(1-\theta)\left\{E\left[\left\|\mathbf{S}^{\varphi}(\mathcal{X})-\mathbf{E}\left(\mathbf{S}^{\varphi}(\mathcal{X})\right)\right\|^{2}\right]+\left\|\mathbf{E}\left(\mathbf{S}^{\varphi}(\mathcal{X})\right)-\mathbf{S}^{\varphi}(\widetilde{U})\right\|^{2}\right\} \\
+\theta\left\{E\left(\left[\rho_{2}^{\varphi}(\mathcal{X}, \widetilde{E}(\mathcal{X}))\right]^{2}\right)+\left[\rho_{2}^{\varphi}(\widetilde{E}(\mathcal{X}), \widetilde{U})\right]^{2}\right\} \\
=\mathfrak{m} \mathfrak{s e}_{\theta}^{\varphi}(\mathcal{X}, \widetilde{E}(\mathcal{X}))+\left[\mathfrak{D}_{\theta}^{\varphi}(\widetilde{E}(\mathcal{X}), \widetilde{U})\right]^{2},
\end{gathered}
$$

whence the result is proved.

As a consequence from the last two propositions, the 'avoidable square error' in estimating the distribution of $\mathcal{X}$ can be quantified when one uses the metric in this paper by means of the square bias $\left[\mathfrak{D}_{\theta}^{\varphi}(\widetilde{E}(\mathcal{X}), \widetilde{U})\right]^{2}$.

Following a similar reasoning, and based on the expressions for the Euclidean norm and the product of elements in $\mathbb{R}^{p}$, one can immediately prove that the support/Steiner-based mean square error of a fuzzy vector value in estimating the value of a random fuzzy vector can also be decomposed in terms of some classical mean square errors as follows: 
Proposition 4.3. Let $\varphi$ be an absolutely continuous probability measure on $\left([0,1], \mathcal{B}_{[0,1]}\right)$ with positive mass function on $(0,1), \theta \in(0,1]$ be a parameter, $\widetilde{U} \in \mathcal{F}_{c}^{*}\left(\mathbb{R}^{p}\right)$ be a fuzzy vector value and $\mathcal{X}$ be a random fuzzy vector associated with the probability space $(\Omega, \mathcal{A}, P)$. If $s_{\mathcal{X}} \in L^{2}(\Omega, \mathcal{A}, P)$ and $\mathbf{u} \cdot s_{\mathcal{X}}(\alpha, \mathbf{u})$ is integrable on $\Omega \times\left([0,1] \times \mathbb{S}^{p-1}\right)$, then

$$
\mathfrak{m} \mathfrak{s e}_{\theta}^{\varphi}(\mathcal{X}, \widetilde{U})=(1-\theta) \sum_{i=1}^{p} \operatorname{MSE}\left(\mathbf{S}^{\varphi}(\mathcal{X}) \cdot \mathbf{e}_{i}, \mathbf{S}^{\varphi}(\widetilde{U}) \cdot \mathbf{e}_{i}\right)+\theta \operatorname{MSE}\left(s_{\mathcal{X}}, s_{\widetilde{U}}\right),
$$

with $\mathbf{e}_{i}$ denoting the $i$-th element in the standard basis for $\mathbb{R}^{p}$, and $\operatorname{MSE}\left(s_{\mathcal{X}}, s_{\widetilde{U}}\right)$ being intended as the classical mean square error of the $\mathbb{H}_{2}$-valued random element $s_{\mathcal{X}}$ about $s_{\widetilde{U}}$ w.r.t. the norm $\|\cdot\|^{\varphi}$ associated with $\rho_{2}^{\varphi}$, that is,

$$
\|f-g\|^{\varphi}=\left[\int_{(0,1]} \int_{\mathbb{S}^{p-1}}[f(\alpha, \mathbf{u})-g(\alpha, \mathbf{u})]^{2} d \lambda_{p}(\mathbf{u}) d \varphi(\alpha)\right]^{1 / 2} .
$$

The remaining part of this section is to be devoted to examine some of the most immediate applications of the use of the mean square error based on the support/Steiner points of random fuzzy vectors.

\subsection{A variance measure of a random fuzzy vector}

The variance of a random element can be introduced by following Fréchet's approach [16], as a measure of the 'least mean square error' in approximating/estimating the values of the random element by a concrete value (or, conversely, the mean error in approximating/estimating this concrete value by means of values of the random element).

The variance of a random fuzzy vector can then be viewed as a measure of how far the corresponding fuzzy vector values are spread out, or how far they are on the average with respect to their (Aumann-type) mean value.

When considering the metric space $\left(\mathcal{F}_{c}^{*}\left(\mathbb{R}^{p}\right), \mathfrak{D}_{\theta}^{\varphi}\right)$ and the Fréchet approach, one can state

Definition 4.5. Given an absolutely continuous probability measure $\varphi$ on $\left([0,1], \mathcal{B}_{[0,1]}\right)$ with positive mass function on $(0,1)$, a parameter $\theta \in(0,1]$, and a random fuzzy vector $\mathcal{X}$ such that $s_{\mathcal{X}} \in L^{2}(\Omega, \mathcal{A}, P)$, the support/Steinerbased variance of $\mathcal{X}$ is the real value given by

$$
\mathfrak{v a r}_{\theta}^{\varphi}(\mathcal{X})=\min _{\widetilde{U} \in \mathcal{F}_{c}^{*}\left(\mathbb{R}^{p}\right)} \mathfrak{m s} \mathfrak{e}_{\theta}^{\varphi}(\mathcal{X}, \widetilde{U})=E\left(\left[\mathfrak{D}_{\theta}^{\varphi}(\mathcal{X}, \widetilde{E}(\mathcal{X}))\right]^{2}\right) .
$$


The support/Steiner-based variance of a random fuzzy vector preserves the most interesting properties of the variance of a real-valued random variable. Thus, following either straightforward arguments for the mean values and variances of real-valued random variables, or arguments similar to those in the proof of Proposition 4.2, we have that

Proposition 4.4. Given an absolutely continuous probability measure $\varphi$ on $\left([0,1], \mathcal{B}_{[0,1]}\right)$ with positive mass function on $(0,1)$, a parameter $\theta \in(0,1]$, and a random fuzzy vector $\mathcal{X}$ associated with the probability space $(\Omega, \mathcal{A}, P)$ and such that $s_{\mathcal{X}} \in L^{2}(\Omega, \mathcal{A}, P)$, then,

i) $\operatorname{var}_{\theta}^{\varphi}(\mathcal{X}) \geq 0$.

ii) $\mathfrak{v a r}_{\theta}^{\varphi}(\mathcal{X})=0$ if, and only if, $\mathcal{X}$ is degenerate at a fuzzy vector value (i.e., $\mathcal{X}$ is almost surely $[P]$ deterministic).

iii) $\mathfrak{v a r}_{\theta}^{\varphi}$ is translational invariant, i.e., $\mathfrak{v a r}_{\theta}^{\varphi}(\mathcal{X}+\widetilde{U})=\mathfrak{v a r}_{\theta}^{\varphi}(\mathcal{X})$ whatever $\widetilde{U} \in \mathcal{F}_{c}^{*}\left(\mathbb{R}^{p}\right)$ may be.

$i v) \mathfrak{v a r}_{\theta}^{\varphi}(\gamma \cdot \mathcal{X})=\gamma^{2} \cdot \mathfrak{v} \mathfrak{a r}{ }_{\theta}^{\varphi}(\mathcal{X})$ whatever $\gamma \in \mathbb{R}$ may be.

Furthermore, if $\mathbf{u} \cdot s_{\mathcal{X}}(\alpha, \mathbf{u})$ is integrable on $\Omega \times\left([0,1] \times \mathbb{S}^{p-1}\right)$, then

$v)$

$$
\mathfrak{v} \mathfrak{r r}_{\theta}^{\varphi}(\mathcal{X})=(1-\theta) \sum_{i=1}^{p} \operatorname{Var}\left(\mathbf{S}^{\varphi}(\mathcal{X}) \cdot \mathbf{e}_{i}\right)+\theta \operatorname{Var}^{\varphi}\left(s_{\mathcal{X}}\right),
$$

with $\operatorname{Var}^{\varphi}\left(s_{\mathcal{X}}\right)$ being intended as the classical variance of an $\mathbb{H}_{2}$-valued random element w.r.t. the norm $\|\cdot\|^{\varphi}$.

vi) If $\mathcal{X}$ and $\mathcal{Y}$ are independent random fuzzy vectors associated with the same probability space, and $\mathbf{u} \cdot s_{\mathcal{Y}}(\alpha, \mathbf{u})$ is integrable on $\Omega \times\left([0,1] \times \mathbb{S}^{p-1}\right)$, then $\mathfrak{v a r}_{\theta}^{\varphi}(\mathcal{X}+\mathcal{Y})=\mathfrak{v a r}_{\theta}^{\varphi}(\mathcal{X})+\mathfrak{v a r}{ }_{\theta}^{\varphi}(\mathcal{Y})$.

\subsection{Estimating the population mean value of a random fuzzy vector}

As for the real-valued case, another immediate application of the support/ Steiner-based mean square error is that of quantifying the goodness of an estimator of a fuzzy vector-valued 'parameter' of the distribution of a fuzzy random vector.

As a simple and useful example for this, one can think in estimating the population mean value of a random fuzzy vector $\mathcal{X}$ on the basis of a simple random sample from it, that is, on the basis of the information supplied by a finite sequence of independent random fuzzy vectors which are identically distributed as $\mathcal{X}$, say $\mathcal{X}_{1}, \ldots, \mathcal{X}_{n}$. We can then state that 
Proposition 4.5. Let $(\Omega, \mathcal{A}, P)$ be a probability space and let $\mathcal{X}$ be a random fuzzy vector associated with this probability space and such that $s_{\mathcal{X}} \in$ $L^{2}(\Omega, \mathcal{A}, P)$. Let $\mathcal{X}_{1}, \ldots, \mathcal{X}_{n}$ be a simple random sample from $\mathcal{X}$. Then,

i) the corresponding sample mean

$$
\overline{\mathcal{X}_{n}}=\frac{1}{n} \cdot\left(\mathcal{X}_{1}+\ldots+\mathcal{X}_{n}\right)
$$

is an unbiased estimator of the population mean $\widetilde{E}(\mathcal{X})$. That is, the mean value of the estimator $\overline{\mathcal{X}_{n}}$ over the space of all samples of $n$ independent observations from $\mathcal{X}$ equals $\widetilde{E}(\mathcal{X})$.

ii) Furthermore, the associated support/Steiner-based mean square error of the fuzzy statistic $\overline{\mathcal{X}_{n}}$ in estimating the value of $\widetilde{E}(\mathcal{X})$ is given by

$$
\mathfrak{m} \mathfrak{s e}_{\theta}^{\varphi}(\overline{\mathcal{X}}, \widetilde{E}(\mathcal{X}))=\frac{\mathfrak{v a r} \mathfrak{r}_{\theta}^{\varphi}(\mathcal{X})}{n}
$$

\subsection{Simulation study}

This subsection aims to illustrate empirically the behaviour of the support/Steiner-based mean square error of a random fuzzy vector about its Aumann-type mean value. The diversity of random fuzzy vectors makes almost unfeasible to think about a simulation study for dimensions greater than 1, so we will constrain our analysis to random fuzzy numbers (i.e., $p=1$ ) by estimating their value through the Aumann-type expected value, that is, by analyzing the behaviour of the corresponding Fréchet variance.

The simulations have been performed following the situations in Sinova et al. [27]. Thus,

Step 1. A sample of size $n=100000$ of trapezoidal fuzzy numbers has been simulated for each of several different situations in such a way that

- to generate the trapezoidal fuzzy data, we have considered four real-valued random variables as follows: $\mathcal{X}=\operatorname{Tra}\left(X_{1}-X_{2}-X_{3}\right.$, $\left.X_{1}-X_{2}, X_{1}+X_{2}, X_{1}+X_{2}+X_{4}\right)$, with

$$
\begin{gathered}
X_{1}=\operatorname{mid} \mathcal{X}_{1}=\left(\inf \mathcal{X}_{1}+\sup \mathcal{X}_{1}\right) / 2, \\
X_{2}=\operatorname{spr} \mathcal{X}_{1}=\left(\sup \mathcal{X}_{1}-\inf \mathcal{X}_{1}\right) / 2, \\
X_{3}=\inf \mathcal{X}_{1}-\inf \mathcal{X}_{0}, X_{4}=\sup \mathcal{X}_{0}-\sup \mathcal{X}_{1} ;
\end{gathered}
$$


- each sample is assumed to be split into a subsample of size $n\left(1-c_{p}\right)$ (where $c_{p}$ denotes the proportion of contamination and is supposed to range in $\{0,0.1,0.2,0.4\}$ ), associated with a non-contaminated distribution, and a subsample of size $n \cdot c_{p}$, associated with a contaminated one. $C_{D}$ plays an additional contamination role, measuring how far the distribution of the contaminated subsample is from the distribution of the non-contaminated one (and ranges in $\{0,1,5,10,100\})$;

- 16 situations for different values of $c_{p}$ and $C_{D}$ have been considered, and for each of them two cases have been selected, namely, one in which the random variables $X_{i}$ are independent (CASE 1) and another one in which they are dependent (CASE 2). More specifically, CASE 1 assumes that:

$\bullet X_{1} \sim \mathcal{N}(0,1)$ and $X_{2}, X_{3}, X_{4} \sim \chi_{1}^{2}$ for the non-contaminated subsample,

$\bullet X_{1} \sim \mathcal{N}(0,3)+C_{D}$ and $X_{2}, X_{3}, X_{4} \sim \chi_{4}^{2}+C_{D}$ for the contaminated subsample,

whereas CASE 2 assumes that:

•• $X_{1} \sim \mathcal{N}(0,1)$ and $X_{2}, X_{3}, X_{4} \sim 1 /\left(X_{1}^{2}+1\right)^{2}+0.1 \cdot \chi_{1}^{2}$ for the non-contaminated subsample (with $\chi_{1}^{2}$ independent of $X_{1}$ ),

•• $X_{1} \sim \mathcal{N}(0,3)+C_{D}$ and $X_{2}, X_{3}, X_{4} \sim 1 /\left(X_{1}^{2}+1\right)^{2}+0.1 \cdot \chi_{1}^{2}+C_{D}$ for the contaminated subsample (with $\chi_{1}^{2}$ independent of $X_{1}$ ).

In any case, the $\chi_{1}^{2}$ are assumed to be independent from $1 /\left(X_{1}^{2}+\right.$ $1)^{2}$.

Step 2. $N=1000$ replications of Step 1 have been considered, so that for each of the 16 situations concerning $c_{p}$ and $c_{D}$ there are 1000 available samples of size $n=100000$.

Step 3. The population Aumann-type mean for the non-contaminated distribution has been approximated by a Monte Carlo approach on the basis of the $1000 \times 100000$ data from the first situation $\left(c_{p}\right.$ $\left.=C_{D}=0\right)$. For each of the 16 situations, the 1000 sample Aumantype means as estimates of the population Aumann-type mean for the non-contaminated distribution are computed. The variance of the estimator has been approximated by the mean squared distance between the mixed sample and the approximate population mean for the non-contaminated distribution. 
Distances have been first computed by considering the $L^{2}$ metric $\mathfrak{D}_{\theta}^{\ell}$. It is convenient to recall that, by particularizing Proposition 4.4.v) to $p=1$, one can easily obtain that

$$
\mathfrak{v a r}_{\theta}^{\varphi}(\mathcal{X})=\operatorname{Var}\left(\operatorname{wabl}^{\varphi} \mathcal{X}\right)+\theta\left[\frac{\operatorname{Var}\left(\operatorname{ldev}_{\mathcal{X}}^{\varphi}\right)+\operatorname{Var}\left(\operatorname{rdev}_{\mathcal{X}}^{\varphi}\right)}{2}\right] .
$$

By using the notations

$$
\begin{gathered}
\operatorname{Var}_{1}=\operatorname{Var}\left(\operatorname{wabl}^{\ell} \mathcal{X}\right), \\
\operatorname{Var}_{2}=\left[\operatorname{Var}^{\ell}\left(\operatorname{ldev}_{\mathcal{X}}^{\ell}\right)+\operatorname{Var}^{\ell}\left(\operatorname{rdev}_{\mathcal{X}}^{\ell}\right)\right] / 2 \\
=\frac{1}{2} \int_{[0,1]} \operatorname{Var}^{\ell}\left(\operatorname{ldev}_{\mathcal{X}}^{\ell}(\alpha)\right) d \ell(\alpha)+\frac{1}{2} \int_{[0,1]} \operatorname{Var}^{\ell}\left(\operatorname{rdev}_{\mathcal{X}}^{\ell}(\alpha)\right) d \ell(\alpha),
\end{gathered}
$$

we have that

$$
\mathfrak{v a r}_{\theta}^{\ell}(\mathcal{X})=\operatorname{Var}_{1}+\theta \operatorname{Var}_{2}
$$

Outputs for this simulation study have been collected in Table 1.

\begin{tabular}{c|c||c|c||c||c||c||c}
\multicolumn{9}{|c|}{} & \multicolumn{9}{c||}{ CASE 1 } & \multicolumn{3}{c}{ CASE 2 } \\
\hline$c_{p}$ & $c_{D}$ & $\operatorname{Var}_{1}$ & $\operatorname{Var}_{2}$ & $\mathfrak{v a r}_{\theta}^{\ell}(\mathcal{X})$ & $\operatorname{Var}_{1}$ & $\operatorname{Var}_{2}$ & $\mathfrak{v a r}_{\theta}^{\ell}(\mathcal{X})$ \\
\hline \hline 0 & 0 & 0.000013 & 0.000024 & $0.000013+0.000024 \theta$ & 0.000010 & 0.000003 & $0.000010+0.000003 \theta$ \\
\hline 0.1 & 0 & 0.000018 & 0.127471 & $0.000018+0.127471 \theta$ & 0.000013 & 0.000224 & $0.000013+0.000224 \theta$ \\
0.1 & 1 & 0.003465 & 0.221472 & $0.003465+0.221472 \theta$ & 0.003318 & 0.011735 & $0.003318+0.011735 \theta$ \\
0.1 & 5 & 0.079310 & 0.923036 & $0.079310+0.923036 \theta$ & 0.079117 & 0.339279 & $0.079117+0.339279 \theta$ \\
0.1 & 10 & 0.334993 & 2.401429 & $0.334993+2.401429 \theta$ & 0.338943 & 1.363894 & $0.338943+1.363894 \theta$ \\
0.1 & 100 & 32.67662 & 153.0057 & $32.67662+153.0057 \theta$ & 33.47193 & 141.5996 & $33.47193+141.5996 \theta$ \\
\hline 0.2 & 0 & 0.000022 & 0.515911 & $0.000022+0.515911 \theta$ & 0.000019 & 0.000965 & $0.000019+0.000965 \theta$ \\
0.2 & 1 & 0.013198 & 0.927163 & $0.013198+0.927163 \theta$ & 0.013293 & 0.046946 & $0.013293+0.046946 \theta$ \\
0.2 & 5 & 0.337334 & 3.648892 & $0.337334+3.648892 \theta$ & 0.324265 & 1.373463 & $0.324265+1.373463 \theta$ \\
0.2 & 10 & 1.356969 & 9.576708 & $1.356969+9.576708 \theta$ & 1.330505 & 5.535713 & $1.330505+5.535713 \theta$ \\
0.2 & 100 & 127.7963 & 616.7394 & $127.7963+616.7394 \theta$ & 130.9568 & 567.5711 & $130.9568+567.5711 \theta$ \\
\hline 0.4 & 0 & 0.000031 & 2.092746 & $0.000031+2.092746 \theta$ & 0.000024 & 0.003353 & $0.000024+0.003353 \theta$ \\
0.4 & 1 & 0.052929 & 3.657314 & $0.052929+3.657314 \theta$ & 0.051238 & 0.189347 & $0.051238+0.189347 \theta$ \\
0.4 & 5 & 1.330964 & 14.69655 & $1.330964+14.69655 \theta$ & 1.352259 & 5.224873 & $1.352259+5.224873 \theta$ \\
0.4 & 10 & 5.257282 & 39.60472 & $5.257282+39.60472 \theta$ & 5.313620 & 21.97364 & $5.313620+21.97364 \theta$ \\
0.4 & 100 & 542.4229 & 2425.986 & $542.4229+2425.986 \theta$ & 534.3272 & 2280.200 & $534.3272+2280.200 \theta$ \\
\hline \hline
\end{tabular}

Table 1: $\mathfrak{D}_{\theta}^{\varphi}$-Variances of the mixed (partially contaminated and noncontaminated) simulated samples from random fuzzy numbers

On the basis of Table 1, one can empirically conclude that the dispersion of the random fuzzy number, when measured in terms of the mean square error about its expected value, is quite sensitive (in the sense of substantially increasing) w.r.t. contamination, especially when the distribution of the contaminated subsample is very far from the distribution of the noncontaminated one (i.e., with large $c_{D}$ ). The dispersion increases with the value of the parameter $\theta$, which weighs the effect of the dispersion in 'shape' (more concretely, in the left and right deviations about the central location). 
On the other hand, it is also interesting to analyze whether the choice of $\mathfrak{D}_{\theta}^{\varphi}$ instead of $D_{\theta}^{\varphi}$ by Trutschnig et al. [33] leads or not to rather different mean square errors, where, in case $p=1$

$$
D_{\theta}^{\varphi}(\widetilde{U}, \widetilde{V})=\sqrt{\int_{[0,1]}\left[\operatorname{mid} \widetilde{U}_{\alpha}-\operatorname{mid} \widetilde{V}_{\alpha}\right]^{2} d \varphi(\alpha)+\theta \int_{[0,1]}\left[\operatorname{spr} \widetilde{U}_{\alpha}-\operatorname{spr} \widetilde{V}_{\alpha}\right]^{2} d \varphi(\alpha)} .
$$

To check the possible influence of such a choice, we take into account that

$$
\begin{gathered}
\operatorname{Var}^{\ell}(\operatorname{mid} \mathcal{X})=\int_{[0,1]} \operatorname{Var}\left(\operatorname{mid} \mathcal{X}_{\alpha}\right) d \ell(\alpha), \operatorname{Var}^{\ell}(\operatorname{spr} \mathcal{X})=\int_{[0,1]} \operatorname{Var}\left(\operatorname{spr} \mathcal{X}_{\alpha}\right) d \ell(\alpha), \\
\operatorname{Var}_{\theta}^{\ell}(\mathcal{X})=\operatorname{Var}^{\ell}(\operatorname{mid} \mathcal{X})+\theta \operatorname{Var}^{\ell}(\operatorname{spr} \mathcal{X}) .
\end{gathered}
$$

Outputs for this simulation study and $D_{\theta}^{\ell}$ have been collected in Table 2 .

\begin{tabular}{c|c||c|c||c|||c||c||c}
\multicolumn{2}{c|}{} & \multicolumn{9}{c||}{ CASE 1 } & \multicolumn{3}{c}{ CASE 2 } \\
\hline$c_{p}$ & $c_{D}$ & $\operatorname{Var}^{\ell}(\mathrm{mid})$ & $\operatorname{Var}^{\ell}(\mathrm{spr})$ & $\operatorname{Var}_{\theta}^{\ell}(\mathcal{X})$ & $\operatorname{Var}^{\ell}(\mathrm{mid})$ & $\operatorname{Var}^{\ell}(\mathrm{spr})$ & $\operatorname{Var}_{\theta}^{\ell}(\mathcal{X})$ \\
\hline \hline 0 & 0 & 0.000014 & 0.000023 & $0.000014+0.000023 \theta$ & 0.000010 & 0.000003 & $0.000010+0.000003 \theta$ \\
\hline 0.1 & 0 & 0.000019 & 0.127470 & $0.000019+0.127470 \theta$ & 0.000013 & 0.000224 & $0.000013+0.000224 \theta$ \\
0.1 & 1 & 0.003466 & 0.221471 & $0.003466+0.221471 \theta$ & 0.003318 & 0.011735 & $0.003318+0.011735 \theta$ \\
0.1 & 5 & 0.079311 & 0.923035 & $0.079311+0.923035 \theta$ & 0.079117 & 0.339279 & $0.079117+0.339279 \theta$ \\
0.1 & 10 & 0.334994 & 2.401428 & $0.334994+2.401428 \theta$ & 0.338943 & 1.363894 & $0.338943+1.363894 \theta$ \\
0.1 & 100 & 32.67661 & 153.0056 & $32.67661+153.0056 \theta$ & 33.47193 & 141.5996 & $33.47193+141.5996 \theta$ \\
\hline 0.2 & 0 & 0.000024 & 0.515910 & $0.000024+0.515910 \theta$ & 0.000019 & 0.000965 & $0.000019+0.000965 \theta$ \\
0.2 & 1 & 0.013199 & 0.927162 & $0.013199+0.927162 \theta$ & 0.013293 & 0.046946 & $0.013293+0.046946 \theta$ \\
0.2 & 5 & 0.337335 & 3.648890 & $0.337335+3.648890 \theta$ & 0.324265 & 1.373463 & $0.324265+1.373463 \theta$ \\
0.2 & 10 & 1.356971 & 9.576707 & $1.356971+9.576707 \theta$ & 1.330505 & 5.535713 & $1.330505+5.535713 \theta$ \\
0.2 & 100 & 127.7962 & 616.7393 & $127.7962+616.7393 \theta$ & 130.9568 & 567.5710 & $130.9568+567.5710 \theta$ \\
\hline 0.4 & 0 & 0.000033 & 2.092744 & $0.000033+2.092744 \theta$ & 0.000024 & 0.003353 & $0.000024+0.003353 \theta$ \\
0.4 & 1 & 0.052931 & 3.657312 & $0.052931+3.657312 \theta$ & 0.051238 & 0.189347 & $0.051238+0.189347 \theta$ \\
0.4 & 5 & 1.330965 & 14.69655 & $1.330965+14.69655 \theta$ & 1.352259 & 5.224872 & $1.352259+5.224872 \theta$ \\
0.4 & 10 & 5.257283 & 39.60472 & $5.257283+39.60472 \theta$ & 5.313621 & 21.97364 & $5.313621+21.97364 \theta$ \\
0.4 & 100 & 542.4229 & 2425.985 & $542.4229+2425.985 \theta$ & 534.3272 & 2280.200 & $534.3272+2280.200 \theta$ \\
\hline \hline
\end{tabular}

Table 2: $D_{\theta}^{\varphi}$-Variances of the mixed (partially contaminated and noncontaminated) simulated samples from random fuzzy numbers

By comparing Tables 1 and 2, one can easily see, regardless the considered perturbations $c_{p}$ and $c_{D}$, that differences between the outputs for $\mathfrak{D}_{\theta}^{\varphi}$ and $D_{\theta}^{\varphi}$ are really small, so there is no essential distinction between them in computing the mean square error (see Table 3 to clearly corroborate this assertion).

Nevertheless, we should emphasize once more that, for theoretical and optimization purposes, there is a clear advantage in favour of $\mathfrak{D}_{\theta}^{\varphi}$ in contrast to $D_{\theta}^{\varphi}$. This is due to the fact that the first one is based on a representation for which one has sufficient conditions characterizing fuzzy vectors, what does not happen for the second one. 


\begin{tabular}{c|c||c||c}
$c_{p}$ & ${ }^{c} D$ & $\mathfrak{v a r}_{\theta}^{\ell}(\mathcal{X})-\operatorname{Var}_{\theta}^{\ell}(\mathcal{X})(\mathbf{C A S E} 1)$ & $\mathfrak{v a r}_{\theta}^{\ell}(\mathcal{X})-\operatorname{Var}_{\theta}^{\ell}(\mathcal{X}) \quad(\mathbf{C A S E} 2)$ \\
\hline \hline 0 & 0 & $.00000083(1-\theta)$ & $.0000000087(1-\theta)$ \\
\hline .1 & 0 & $.00000109(1-\theta)$ & $.0000000083(1-\theta)$ \\
.1 & 1 & $.00000100(1-\theta)$ & $.0000000093(1-\theta)$ \\
.1 & 5 & $.00000105(1-\theta)$ & $.0000000084(1-\theta)$ \\
.1 & 10 & $.00000092(1-\theta)$ & $.0000000086(1-\theta)$ \\
.1 & 100 & $.00000102(1-\theta)$ & $.0000000087(1-\theta)$ \\
\hline .2 & 0 & $.00000120(1-\theta)$ & $.000000000086(1-\theta)$ \\
.2 & 1 & $.00000124(1-\theta)$ & $.0000000080(1-\theta)$ \\
.2 & 5 & $.00000118(1-\theta)$ & $.0000000088(1-\theta)$ \\
.2 & 10 & $.00000122(1-\theta)$ & $.000000000000000089(1-\theta)$ \\
.2 & 100 & $.00000131(1-\theta)$ & $.0000000084(1-\theta)$ \\
\hline .4 & 0 & $.00000164(1-\theta)$ & $.0000000091(1-\theta)$ \\
.4 & 1 & $.00000166(1-\theta)$ & $.0000000084(1-\theta)$ \\
.4 & 5 & $.00000173(1-\theta)$ & $.0000000083(1-\theta)$ \\
.4 & 10 & $.00000170(1-\theta)$ & $.00000157(1-\theta)$
\end{tabular}

Table 3: Comparative simulation study on the difference between the sample variances of random fuzzy numbers based on two different $L^{2}$ metrics

\section{Concluding remarks}

In this paper, a parameterized $L^{2}$ metric between fuzzy vectors has been introduced. The metric shows the following two main features:

- its weight parameter allows weighing the effect of the deviation in central location in contrast to the deviation in shape, and

- it is based on a representation of fuzzy vectors for which there exist sufficient conditions for characterizing fuzzy vectors.

Furthermore, simulation developments have shown that it behaves in a very similar way to a previous metric by Bertoluzza et al. [3] (see Trutschnig et al. [33] for the $p$-dimensional version), with the added value of the highlighted second feature which is not fulfilled for Bertoluzza et al.'s distance.

The decomposability of the mean square error based on this metric, along with its properties and features, will be useful in future applications. Among them, it would be interesting to consider how it can be adapted to deal with regression problems involving fuzzy random vectors.

\section{Acknowledgements}

The authors are deeply grateful to the reviewers and Guest Editors handling the original of this paper. Their valuable and insightful suggestions and the meticulous proofreading have been very helpful for the revision. The research in this paper has been partially supported by the Grants from the Spanish Ministry of Education and Science MTM2009-09440-C02-01, the 
Principality of Asturias Grant SV-PA-13-ECOEMP-66, as well as the FPU Grant AP2009-1197 (Sinova) and the Severo Ochoa-Principality of Asturias Grant BP12012 (de la Rosa de Sáa). Their financial support is gratefully acknowledged.

\section{References}

[1] G. Aletti, E.G. Bongiorno, A decomposition theorem for fuzzy set-valued random variables, Fuzzy Set Syst. 219 (2013) 98-112.

[2] I. Beg, S. Ashraf, Simmilarity measures for fuzzy sets, Appl. and Comput. Math., 8(2) (2009) 192-202.

[3] C. Bertoluzza, N. Corral, A. Salas, On a new class of distances between fuzzy numbers, Math. Soft. Comput. 2 (1995) 71-84.

[4] A. Blanco-Fernández, M.R. Casals, A. Colubi, N. Corral, M. García-Bárzana, M.A. Gil, G., González-Rodríguez, M.T. López, M.A. Lubiano, M. Montenegro, A.B. Ramos-Guajardo, S. de la Rosa de Sáa, B. Sinova, A distance-based statistical analysis of fuzzy number-valued data, Int. J. Approx. Reas. 55 (2014) 1487-1501.

[5] A. Blanco-Fernández, M.R. Casals, A. Colubi, N. Corral, M. García-Bárzana, M.A. Gil, G., González-Rodríguez, M.T. López, M.A. Lubiano, M. Montenegro, A.B. Ramos-Guajardo, S. de la Rosa de Sáa, B. Sinova, Rejoinder on "A distance-based statistical analysis of fuzzy number-valued data", Int. J. Approx. Reas. 55 (2014) 1601-1605.

[6] D. Butnariu, M. Navara, M., T. Vetterlein, Linear space of fuzzy vectors, in: S. Gottwald, P. Hájek, U. Höhle., E.P. Klement (Eds.), Fuzzy Logics and Related Structures, Linz, 2005, pp. 23-26.

[7] M.R. Casals, N. Corral, M.A. Gil, M.T. López, M.A. Lubiano, M. Montenegro, G. Naval, A. Salas, Bertoluzza et al.'s metric as a basis for analyzing fuzzy data, Metron 71(3) (2013) 307-322.

[8] C. Castaing, M. Valadier, Convex Analysis and Measurable Multifunctions. Lec. Notes in Math. 580, Springer-Verlag, Berlin, 1977.

[9] L. de Campos, A. González, A subjective approach for ranking fuzzy numbers, Fuzzy Set Syst. 29 (1989) 145-153.

[10] P. Diamond, P. Kloeden, Metric spaces of fuzzy sets, Fuzzy Set Syst. 35 (1990) 241-249.

[11] P. Diamond, P. Kloeden, The parametrization of fuzzy sets by single-valued mappings, in: R. Lowen, M. Roubens (Eds.), Fuzzy Logic, Theory and Decision Library 12, Springer (formerly Kluwer), Dordrecht, 1993, pp. 95-101. 
[12] P. Diamond, P. Kloeden, Metric Spaces of Fuzzy Sets: Theory and Applications, World Scientific, Singapore, 1994.

[13] P. Diamond, R. Körner, Extended fuzzy linear models and least squares estimates, Computers Math. Applic. 33(9) (1997) 15-32.

[14] D. Dubois, H. Prade, F. esteva, P. García, L. Godo, A logical approach to interpolation based on similarity relations, Int. J. Approx. Reason. 17 (1997) 1-36.

[15] F. Esteva, L. Godo, R.O. Rodríguez, T. Vetterlein, Logics for approximate and strong entailments, Fuzzy Sets Syst. 197 (2012) 59-70.

[16] M. Fréchet, Les éléments aléatoires de nature quelconque dans un espace distancié, Ann. L'Inst. H. Poincaré 10 (1948) 215-310.

[17] P.K. Ghosh, K.V. Kumar, Support function representation of convex bodies, its application in geometric computing, and some related representations, Comp. Vis. Im. Under. 72(3) (1998) 379-403.

[18] G. González-Rodríguez, A. Colubi, M.A. Gil, Fuzzy data treated as functional data: A one-way ANOVA test approach, Comput. Stat. Data An. 56 (2012) 943-955.

[19] E.P. Klement, M.L. Puri, D.A. Ralescu, Limit theorems for fuzzy random variables, Proc. R. Soc. Lond. A 407 (1986) 171-182.

[20] R. Körner, On the variance of fuzzy random variables, Fuzzy Set Syst. 92 (1997), 83-93.

[21] J. Liang, M. Navara, T. Vetterlein, Different representations of fuzzy vectors, in: C. Sossai, G. Chemello (Eds.), Symbolic and Quantitative Approaches to Reasoning with Uncertainty, Lecture Notes in Computer Sciences 5590, Springer, Berlin, 2005, pp. 700-711.

[22] E.N. Nasibov, To linear transformations with fuzzy arguments, Trans. Acad. Sciences Azerbaijan, Ser. Phys-tecn. and Math. Sciences, 6 (1989) 164-169.

[23] H.T. Nguyen, A note on the extension principle for fuzzy sets, J. Math. Anal. Appl., 64 (1978) 369-380.

[24] M.L. Puri, D.A. Ralescu, The concept of normality for fuzzy random variables, Ann. Probab. 11 (1985) 1373-1379.

[25] M.L. Puri, D.A. Ralescu, Fuzzy random variables, J. Math. Anal. Appl. 114 (1986) 409-422.

[26] R. Schneider, On Steiner points of convex bodies. Israel J. Math., 9 (1971) 241-249.

[27] B. Sinova, M.A. Gil, A. Colubi, S. Van Aelst, The median of a random fuzzy number. The 1-norm distance approach, Fuzzy Sets Syst. 200 (2012) 99-115. 
[28] B. Sinova, M.A. Gil, M.T. López, S. Van Aelst, A parameterized $L^{2}$ metric between fuzzy numbers and its parameter interpretation, Fuzzy Sets Syst. 245 (2014) 101-115.

[29] N. Sugano, Fuzzy set theoretical approach to a chromatic relevant color on the natural color system, Int. J. Innov. Comp., Inform. Contr. 2(1) (2006) 193-203.

[30] N. Sugano, Color-naming system using fuzzy set theoretical approach, IEEE Int. Conf. Fuzzy Syst. 1 (2001) 81-84.

[31] N. Sugano, Fuzzy set theoretical approach to the tone triangular system, J. Computers 6(11) (2011) 2345-2356.

[32] N. Sugano, S. Komatsuzaki, H. Ono, Y. Chiba, Fuzzy set theoretical analysis of human membership values on the color triangle, J. Computers 4(7) (2009) 593-600.

[33] W. Trutschnig, G. González-Rodríguez, A. Colubi, M.A. Gil, A new family of metrics for compact, convex (fuzzy) sets based on a generalized concept of mid and spread. Inform. Sciences 179 (2009) 3964-3972.

[34] T. Vetterlein, M. Navara, The Steiner centroid of fuzzy sets, in: Proc. IFSA'2005, Pekin, 2005, pp. 1256-1258.

[35] T. Vetterlein, M. Navara, Defuzzification using Steiner points, Fuzzy Set Syst. 157 (2006) 1455-1462.

[36] R.R. Yager, A procedure for ordering fuzzy subsets of the unit interval, Inform. Sciences 24 (1981) 143-161.

[37] L.A. Zadeh, The concept of a linguistic variable and its application to approximate reasoning, Part 1, Inform. Sci., 8 (1975) 199-249; Part 2, Inform. Sci., 8 (1975) 301-353; Part 3, Inform. Sci., 9 (1975) 43-80. 